\title{
Retinal amyloid pathology and proof-of-concept imaging trial in Alzheimer's disease
}

Yosef Koronyo, ${ }^{1}$ David Biggs, ${ }^{2}$ Ernesto Barron, ${ }^{3}$ David S. Boyer, ${ }^{4}$ Joel A. Pearlman, ${ }^{5}$ William J. Au, ${ }^{6}$ Shawn J. Kile, ${ }^{6}$ Austin Blanco, ${ }^{2}$ Dieu-Trang Fuchs, ${ }^{1}$ Adeel Ashfaq, ${ }^{7}$ Sally Frautschy, ${ }^{8}$ Gregory M. Cole, ${ }^{8}$ Carol A. Miller, ${ }^{9}$ David R. Hinton, ${ }^{10}$ Steven R. Verdooner, ${ }^{2}$ Keith L. Black, ${ }^{1}$ and Maya Koronyo-Hamaoui,11

'Department of Neurosurgery, Maxine Dunitz Neurosurgical Research Institute, Cedars-Sinai Medical Center, Los Angeles, California, USA. ${ }^{2}$ NeuroVision Imaging LLC, Sacramento, California, USA. ${ }^{3}$ Doheny Eye Institute, Los Angeles, California, USA. ${ }^{4}$ Retina Vitreous Associates Medical Group, Beverly Hills, California, USA. ${ }^{5}$ Retinal Consultants Medical Group, Sacramento, California, USA. ${ }^{6}$ Sutter Neuroscience Institute, Sacramento, California, USA. ${ }^{7}$ David Geffen School of Medicine, University of California, Los Angeles, Los Angeles, California, USA. ${ }^{8}$ Departments of Neurology and Medicine, University of California, Los Angeles, Los Angeles, California, USA; Geriatric Research Education and Clinical Center, Los Angeles, California, USA; and Veterans Greater Los Angeles Healthcare System, Los Angeles, California, USA. ${ }^{9}$ Department of Pathology Program in Neuroscience, Keck School of Medicine, and ${ }^{10}$ Departments of Pathology and Ophthalmology, USC Roski Eye Institute, Keck School of Medicine, University of Southern California, Los Angeles, California, USA. "Department of Biomedical Sciences, Cedars-Sinai Medical Center, Los Angeles, California, USA.

BACKGROUND. Noninvasive detection of Alzheimer's disease (AD) with high specificity and sensitivity can greatly facilitate identification of at-risk populations for earlier, more effective intervention. $A D$ patients exhibit a myriad of retinal pathologies, including hallmark amyloid $\beta$-protein (A $\beta$ ) deposits.

METHODS. Burden, distribution, cellular layer, and structure of retinal A $\beta$ plaques were analyzed in flat mounts and cross sections of definite AD patients and controls $(n=37)$. In a proof-of-concept retinal imaging trial $(n=16)$, amyloid probe curcumin formulation was determined and protocol was established for retinal amyloid imaging in live patients.

RESULTS. Histological examination uncovered classical and neuritic-like $A \beta$ deposits with increased retinal $A \beta_{42}$ plaques (4.7-fold; $\left.P=0.0063\right)$ and neuronal loss $(P=0.0023)$ in $A D$ patients versus matched controls. Retinal $A \beta$ plaque mirrored brain pathology, especially in the primary visual cortex $(P=0.0097$ to $P=0.0018$; Pearson's $r=0.84-0.91)$. Retinal deposits often associated with blood vessels and occurred in hot spot peripheral regions of the superior quadrant and innermost retinal layers. Transmission electron microscopy revealed retinal A $\beta$ assembled into protofibrils and fibrils. Moreover, the ability to image retinal amyloid deposits with solid-lipid curcumin and a modified scanning laser ophthalmoscope was demonstrated in live patients. A fully automated calculation of the retinal amyloid index (RAI), a quantitative measure of increased curcumin

Conflict of interest: $Y$. Koronyo, K.L. Black, S.R. Verdooner, and M. Koronyo-Hamaoui are founding members of NeuroVision Imaging. S. Frautschy and G.M. Cole are founding members of Optimized Curcumin Longvida.

Submitted: February 23, 2017

Accepted: July 7, 2017

Published: August 17, 2017

\section{Reference information:}

JCI Insight. 2017;2(16):e93621. https://doi.org/10.1172/jici.

insight.93621. fluorescence, was constructed. Analysis of RAI scores showed a 2.1-fold increase in AD patients versus controls $(P=0.0031)$.

CONCLUSION. The geometric distribution and increased burden of retinal amyloid pathology in $A D$, together with the feasibility to noninvasively detect discrete retinal amyloid deposits in living patients, may lead to a practical approach for large-scale AD diagnosis and monitoring.

FUNDING. National Institute on Aging award (AG044897) and The Saban and The Marciano Family Foundations. 


\section{Introduction}

Alzheimer's disease (AD) is an invariably fatal neurodegenerative disorder and the leading cause of senile dementia worldwide (1). There is no effective treatment and limited functionality for early unequivocal diagnosis. Aggregation and propagation of misfolded amyloid $\beta$-protein $(\mathrm{A} \beta)$ and hyperphosphorylated tau protein (pTau) present in neurofibrillary tangles (NFTs) are pathological hallmarks that distinctly contribute to $\mathrm{AD}$ diagnosis $(2,3)$. Cerebral $\mathrm{A} \beta$ accumulation may occur as early as 20 years before the onset of clinical dementia, an insidious phase denoted as prodromal AD (4-6). With the development of promising disease-modifying therapies for $\mathrm{AD}$, intervention during the prodromal phase, when damage to synapses and neuronal tissue is minimal, would offer significantly increased treatment efficacy. Existing brain amyloid-imaging tools are invaluable for research and diagnosis (7), yet they present challenges for screening large-scale populations in clinical settings and predicting disease progression $(8,9)$. In part, this is due to high costs, limited availability, and exposure to radioactive isotopes (8-10). These factors prompted the investigation of amyloid pathology in the retina, a CNS tissue readily accessible for direct, noninvasive imaging at high resolution and low cost, to define at-risk populations for further clinical evaluation with gold-standard brain imaging.

Mounting evidence indicates that patients with $\mathrm{AD}$ and mild cognitive impairment (MCI) exhibit a wide spectrum of ocular abnormalities, circadian rhythm disturbances, and visual impairments (reviewed in Hart et al., ref. 11). In addition to afflicting the brain, $\mathrm{AD}$ also affects the retina, a developmental outgrowth of the brain (12-14), possibly causing these symptoms. The retinas of AD patients display pathologies such as nerve fiber layer (NFL) thinning, retinal ganglion cell (RGC) degeneration, reduction of blood flow and vascular alterations, astrogliosis, and abnormal electroretinogram patterns (11, 15-33). Among the many characteristics it shares with the brain, the retina contains neurons, astroglia, microglia, microvasculature with similar morphological and physiological properties, and a blood barrier (12-14, 34, 35). Axons of the optic nerve connect the retina and brain directly and facilitate vesicular transportation of amyloid precursor protein (APP) synthesized in RGCs (36). Further, retinal neurons and glia express proteins that have been implicated in the amyloid cascade (e.g., BACE1, $\gamma$-secretase, APOE) $(35,37,38)$.

While the hallmark pathologies of $\mathrm{AD}$ have long been established in the brain, their manifestation in the human retina is a recent finding $(11,26,39-42)$. We previously identified $A \beta$ plaque pathology in postmortem retinas of definite AD patients as well as in early-stage cases (40). Neuropathological examination revealed that retinal $\mathrm{A} \beta$ plaque burden qualitatively corresponded to cerebral amyloid burden in these patients, in stark contrast to the minimal A $\beta$ plaque findings in age-matched healthy controls $(26,40)$. Subsequent studies have corroborated these findings by demonstrating the existence of retinal A $\beta$ deposits, identifying increased $A \beta_{1-42}$ peptides in the human retina by biochemical analysis (albeit to a lower extent than those seen in neocortical tissues), and, moreover, showing the presence of pTau in retinal tissues of $\mathrm{AD}$ patients $(39,41,42)$. Although a few studies were unable to detect $\mathrm{A} \beta(41,43)$ or pTau $(43)$ in the human $\mathrm{AD}$ retina, several independent groups confirmed their existence by applying specific tissue processing and immunostaining protocols and screening large retinal regions that had previously been overlooked $(11,26,39-42)$. In a recent study, notable $A \beta$ accumulation inside and around degenerating melanopsin-containing RGCs (mRGCs), photoreceptors known to drive circadian photoentrainment, was found in $\mathrm{AD}$ patients (26). The presence of $\mathrm{A} \beta$ may be responsible for loss of this photoreceptor subtype and could possibly explain sleep disturbances seen in these patients (44).

In line with the above findings, numerous studies examining the retinas of sporadic and transgenic animal models of $\mathrm{AD}$ have reported $\mathrm{A} \beta$ deposits, vascular $\mathrm{A} \beta$, pTau, and paired helical filament-tau (PHF-tau), often in association with RGC degeneration, local inflammation (i.e., microglial activation), and impairments of retinal structure and function $(11,39,40,42,45-61)$. These studies, which included a variety of transgenic rat and mouse models, as well as the sporadic rodent model of $\mathrm{AD}$, Octodon degus, demonstrated abundant $A \beta$ deposits, mainly in the innermost retinal layers (RGCs and NFL) (40, 42, $45,49,52,54,57)$. Furthermore, several publications, including ours, have reported a positive response to therapy in reducing retinal $\mathrm{A} \beta$ plaque burden in transgenic (ADtg) murine models, often reflecting the reaction observed in the respective brains $(40,48,51,52,56,60)$. To visualize retinal A $\beta$ pathology in vivo, we had previously developed a noninvasive retinal amyloid imaging method for rodent ADtg models, using curcumin as a contrast agent $(40,51)$. Curcumin is a natural and safe phytopolylphenol fluorochrome that binds to A $\beta$ fibrils and oligomers with high affinity and specificity (62-68). This approach enabled noninvasive detection and monitoring of individual retinal $\mathrm{A} \beta$ deposits in live $\mathrm{ADtg}$ mice $(40,51)$. 

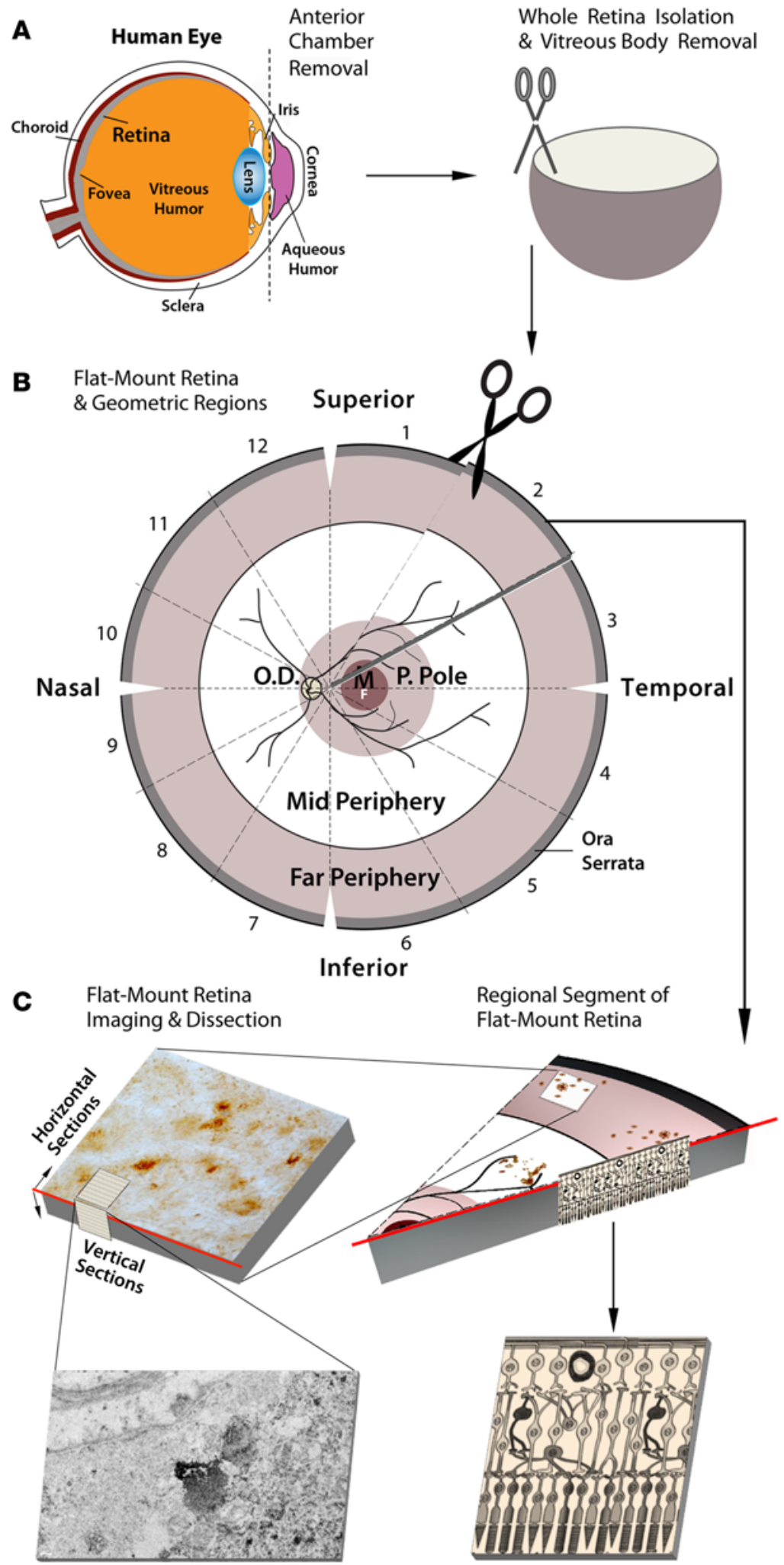

Transmission Electron Microscopy Cross Sectioning \& Imaging
Retinal Cross Sectioning \& Imaging
Figure 1. Schematic diagram of donor eye dissection, retinal isolation, and tissue processing for histological analysis. (A) Anterior chamber extraction from human donor eyeballs, isolation of whole retinas, and vitreous body removal ( $n=37 \mathrm{AD}$ patients and controls, Supplemental Table 1). (B) Preparation of retinal flat mounts and sectioning into 12 geometric regions. (C) Representative segmentation and imaging of regional flat mount stained for $A \beta$ (top right). Region dissection of $A \beta$-positive areas and preparation of horizontal (en face) and vertical (cross) sections for transmission electron microscopy (TEM) analysis (top left). Microscopic image shows a vertical TEM image with ultrastructure of retinal $A \beta$ plaque in a confirmed $A D$ patient (bottom left). Illustration of paraffin-embedded retinal cross section prepared from $A \beta$-immunoreactive areas, showing cellular layers (bottom right). P. Pole, posterior pole; O.D., optic disc; M, macula; F, fovea.

Here, we investigated the burden and geometrical and layer distribution of retinal $A \beta$ deposits and their ultrastructures in definite $\mathrm{AD}$ patients and healthy controls. In a subset of human samples, we quantitatively assessed the correlation between retinal and cerebral plaques and coexistence of retinal neuronal loss. These findings supported translation of our retinal optical imaging approach to humans, which demonstrated the feasibility to detect and quantify retinal amyloid deposits in living patients in a proof-of-concept human trial.

\section{Results}

Geometric distribution of retinal $A \beta$ deposits in $A D$ patients. Eyes and brains were collected from 23 clinically and neuropathologically confirmed $\mathrm{AD}$ patients (mean age \pm SEM: $78.8 \pm 2.9$ years; range 48-98 years; 14 females and 9 males) and 14 healthy controls (mean age \pm SEM: $76.7 \pm 3.4$ years; range: $58-95$ years; 6 females and 8 males), as detailed in Supplemental Table 1 (supplemental material available online with this article; https://doi.org/10.1172/jci.insight.93621DS1). Retinas were isolated and processed as illustrated in Figure 1. Retinal flat mounts and cross sections were stained with various anti-A $\beta$ compounds and mAbs $(12 \mathrm{~F} 4$, $6 \mathrm{E} 10,4 \mathrm{G} 8,11 \mathrm{~A} 5-\mathrm{B} 10)$ using peroxidase- and fluorescent-based techniques. In all $\mathrm{AD}$ cases, we observed increased retinal $\mathrm{A} \beta$ immunoreactivity and deposits compared with age- and sex-matched controls (Figures 2-4 and Supplemental Figure 1).

Analysis of the superior quadrants stained with $12 \mathrm{~F} 4 \mathrm{mAb}$ (specific to $\mathrm{C}^{\prime}$-terminus of $\mathrm{A} \beta_{42}$ species) revealed clusters of $A \beta$ deposits in the retinas of $A D$ patients (Figure $2 \mathrm{~A}$ ) versus their age- and sex-matched controls (Figure 2A). The representative subset of patients and matched controls were aged 60-90 years

(Figure 2A; most of this subset was also analyzed in other retinal regions in La Morgia et al., ref. 26). Control individuals occasionally showed sparse and diffuse retinal $\mathrm{A} \beta$ deposits (Figure $2 \mathrm{~A}$ ), in accordance with their brain pathology. High-magnification images show diverse types of $A \beta$ deposits in $A D$ patient retinas, 
A
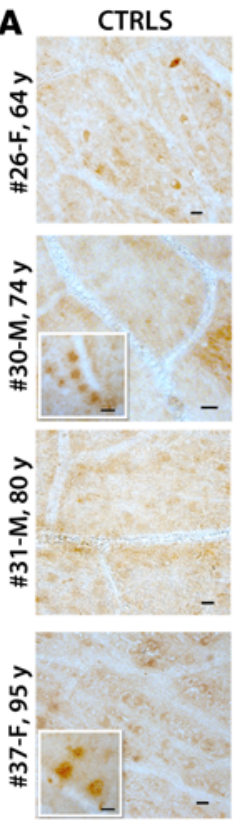

12F4 - DAB
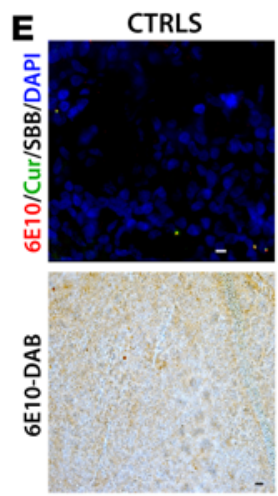

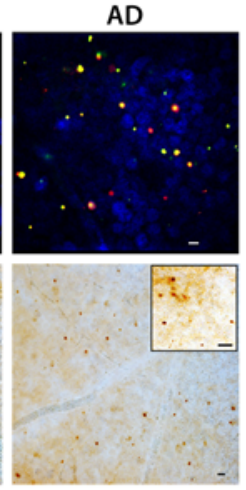

$A D$
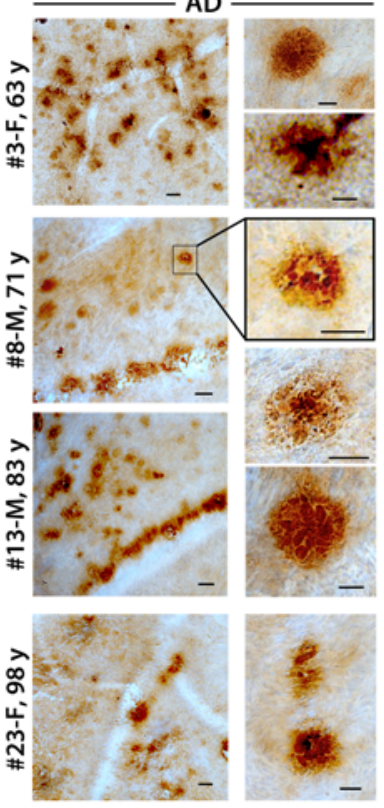

$A D$

F
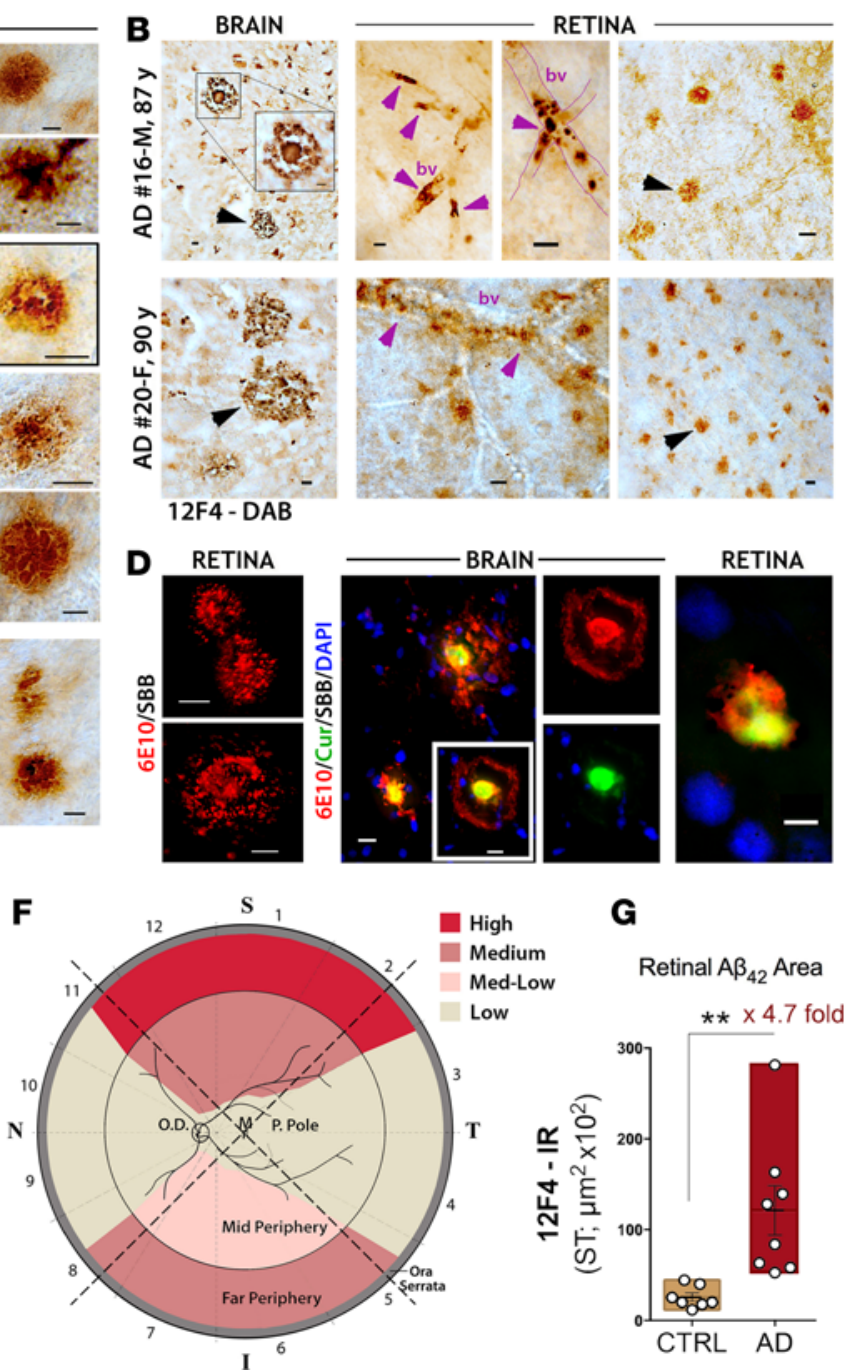

G

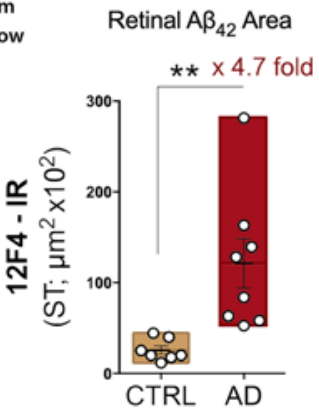

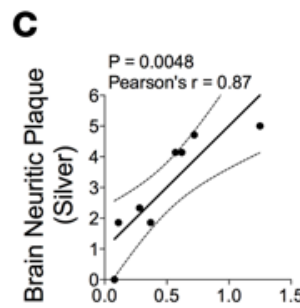
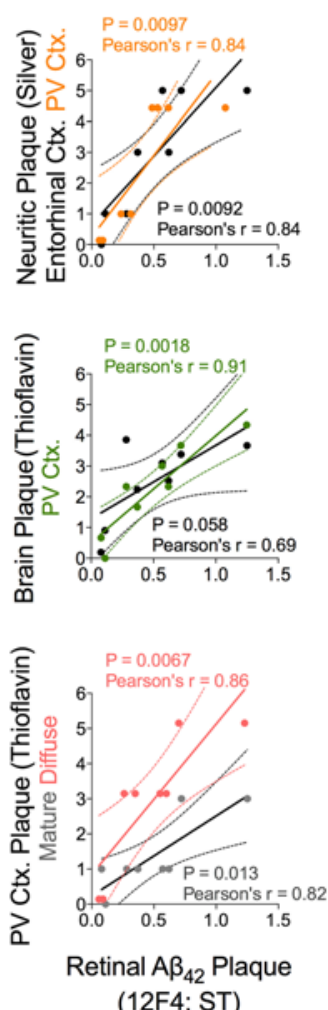

(12F4; ST)

Figure 2. Burden and distribution of $A \beta$ deposits in postmortem retinas of $A D$ patients. (A) Representative micrographs from flat-mount retinas of definite $A D$ patients $(n=8)$ and age- and sex-matched non-AD controls (CTRLs; $n=7)$ at varying ages, stained with anti-A $\beta_{42}$ mAb (12F4) and the standard peroxidase technique (plaques visible as dark brown spots). Scale bar: $20 \mu \mathrm{m}$. Human donor number, sex (male [M] or female [F]), and age in years (y) are shown (for additional details on donor eyes and brains, see Supplemental Table 1). High-magnification images display diverse morphology of $A \beta$ aggregates, including diffuse, compact, and "classical" mature plaques. Scale bar: $10 \mu \mathrm{m}$. (B) AD patients brain sections and their respective retinal flat mounts stained with $12 \mathrm{~F} 4 \mathrm{mAb}$. Retinal plaques are generally smaller but similar in morphology to brain plaques (arrowheads). Retinal A $\beta$ deposits are apparent inside blood vessels (bv), perivascular, and along the vessel walls (purple arrowheads). Scale bar: $20 \mu \mathrm{m}$. (C) Correlation analyses using Pearson's correlation coefficient $(r)$ test between retinal 12F4+-plaque burden and cerebral plaques (Gallyas silver or thioflavin-S staining), including mean plaque burden from 7 brain regions (see Methods), primary visual (PV) cortex (Ctx.) only, and entorhinal Ctx. only, in a subset of AD patients and CTRLs ( $n=8$ ). (D) Anti-A $\beta$ antibody and Longvida curcumin (Cur) fluorescent staining of $A \beta$ deposits in cortical and retinal tissues from the same patient subset. Sudan black B (SBB) was applied to quench autofluorescence. Retinal $A \beta$ plaques positive for $6 E 10$ single staining. Colocalization of curcumin and $6 E 10$ in cortical and retinal $A \beta$ plaques, showing patterns unique to each staining method. Scale bar: $10 \mu \mathrm{m}$ (left retina and brain); $5 \mu \mathrm{m}$ (right retina). (B and $\mathbf{D})$ Representative paired retinal and brain samples from $n=5 \mathrm{AD}$ patients and $n=2$ controls. (E) Distribution of plaques in large retinal regions ( $n=16 \mathrm{AD}$ patients and controls), using fluorescent-based (top; scale bar: $5 \mu \mathrm{m}$ ) and peroxidase-based (bottom; scale bar: $20 \mu \mathrm{m}$ ) staining. (F) Qualitative geometric hot spot regions for retinal $A \beta$ deposits found in $A D$ patients ( $\mathrm{S}$, superior; $\mathrm{T}$, temporal; I, inferior; $\mathrm{N}$, nasal; cumulative data from multiple experiments). (G) Quantitative 12F4-immunoreactive (IR) area of $A \beta_{42}$-containing plaques (geometric regions ST1-3) in a subset of definite AD patients $(n=8)$ and age- and sex-matched controls $(n=7)$. Group mean and SEM are shown. ${ }^{* *} P<0.01$, unpaired 2-tailed Student's $t$ test.

including classical, diffuse, and compact plaques (Figure 2A and Supplemental Figure 1, A and B). The classic $\mathrm{A} \beta$ plaque morphology often seen in $\mathrm{AD}$ patient brains (Figure $2 \mathrm{~B}$, left top image) was also detected in the retinas (e.g., in the 71-year-old male in Figure 2A, right boxed image). A comparison between retinas and respective brains from two additional patients (cases 16 and 20, Supplemental Table 1) indicated resemblance in $\mathrm{A} \beta$ plaque morphology (Figure $2 \mathrm{~B}$, arrowheads). These patients were diagnosed with cerebral amyloid angiopathy and exhibited retinal $\mathrm{A} \beta$ deposits associated with vasculature (Figure $2 \mathrm{~B}$, purple 
A

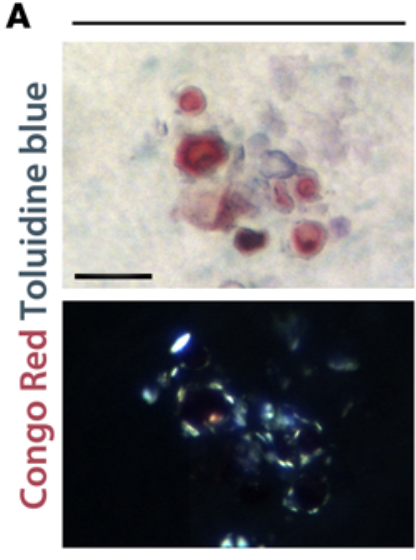

$A D$

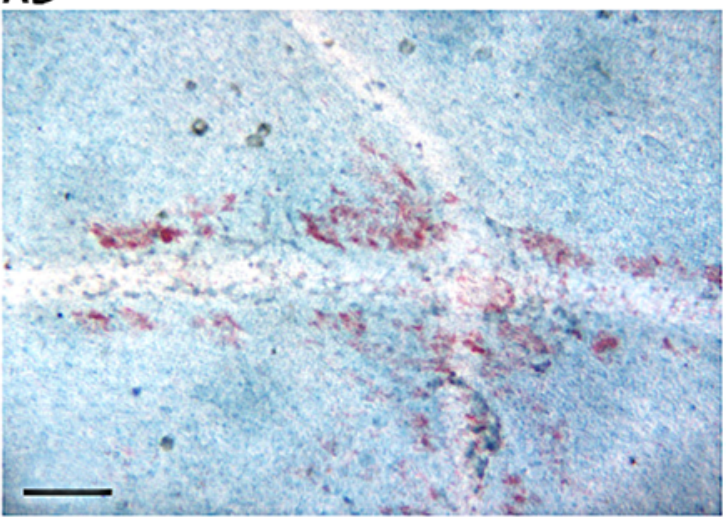

B
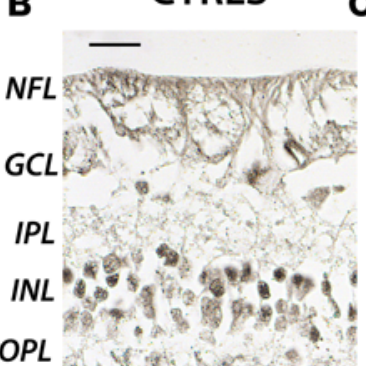

OPL
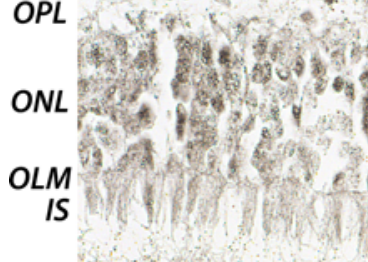

D

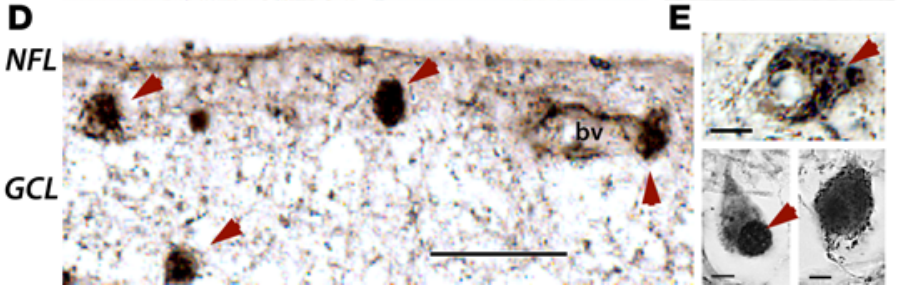

AD
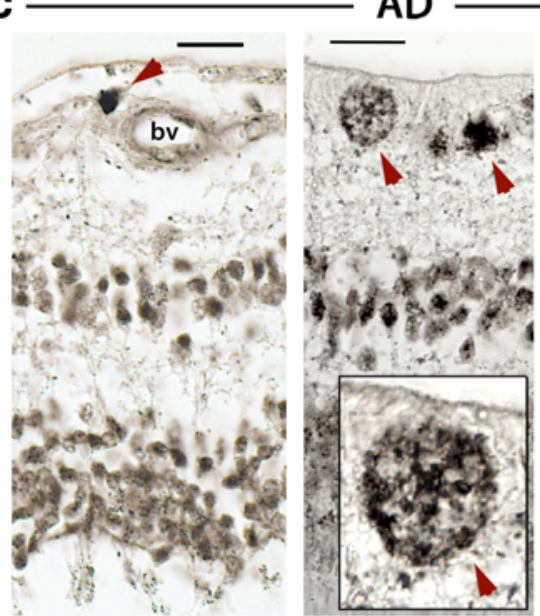

E

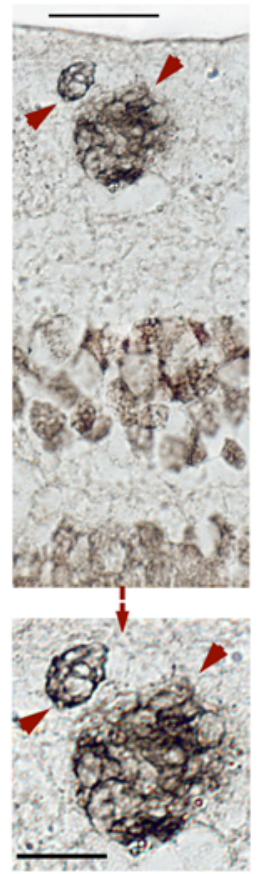

Figure 3. Congo red-positive amyloid fibrils and Gallyas silver-stained neuritic-like plaques found in retinas of $A D$ patients.

(A) Amyloid deposits stained with Congo red detected in flat-mount retinas of AD patients (top left, $n=3$ ). Birefringence (apple-green) of Congo red-stained retinas under polarized light indicates presence of amyloid fibrils (bottom left). Scale bar: $25 \mu \mathrm{m}$. Congo red-positive amyloid plaques in $\mathrm{AD}$ patient retinas (right). Scale bar: $100 \mu \mathrm{m}$. Two intersecting blood vessels surrounded (along blood vessels and perivascular) by extensive Congo red-positive staining; matched control retina was negative (data not shown). Background blue counterstain is Toluidine blue. (B-E) Gallyas silver stain in Paraffin-embedded retinal cross sections from AD patients $(n=12)$ and matched CTRLs $(n=8)$. Scale bar: $20 \mu \mathrm{m}$, unless indicated otherwise. (B) Age-matched controls exhibited intact retinal tissue that lacked major protein aggregates. (C) AD-associated neuropathologies in the retina (red arrowheads), notably observed in the ganglion cell layer (GCL). A compact plaque near a blood vessel (bv; left). A classic $A \beta$ plaque and compact deposits (middle); a higher-magnification image is shown in the inset. Neuritic components of senile plaques in GCL (right); a higher-magnification image is shown below (scale bar: $10 \mu \mathrm{m}$ ). (D) Retinal deposits in GCL near and surrounding a blood vessel. (E) Intracellular/soma-positive silver stain aggregates and nuclear-dominant silver stain (red arrowheads) are observed in GCL and INL (scale bar: $5 \mu \mathrm{m}$ ).

arrowheads), surrounding (perivascular), along, or within blood vessel walls. Retinal A $\beta$ plaques were morphologically similar to their cerebral counterparts, but their diameter was 6 to 7 times smaller on average (Figure $2 \mathrm{~B}$, black arrowheads). In a subset of $\mathrm{AD}$ patients and controls, correlation analyses between retinal $\mathrm{A} \beta_{42}$ plaque and cerebral neuritic plaque burden (silver stain) in various brain regions showed significant and positive linear associations, especially in the entorhinal and primary visual cortex regions (Figure 2C; $P$ $<0.01$ to $P<0.005$, by Pearson's correlation coefficient $[r]$ test). While retinal A $\beta_{42}$ plaques in the superior temporal quadrant showed a trend of association or no association with thioflavin- $\mathrm{S}^{+}$plaques in certain brain regions, there was a consistently strong correlation to thioflavin- $\mathrm{S}^{+}$diffuse, immature, and mature plaques in the primary visual cortex (Figure 2C and Supplemental Figure 2).

Next, we analyzed curcumin fluorescence patterns when bound to retinal and brain A $\beta$ deposits (Figure 2D) using $6 \mathrm{E} 10 \mathrm{mAb}$ alone or in combination with lipidated curcumin (Longvida), a tracer further used for live imaging. Figure 2D displays retinal A $\beta$ plaques of classical morphology using single-antibody labeling, which was comparable to the peroxidase-based staining patterns shown in retinas and brains (Figure 2, A and $\mathrm{B}$, images highlighted by boxed regions). Curcumin labeled the central dense $\mathrm{A} \beta$ core intensely and, to a lesser degree, the radiating fibril arms (Figure 2D). Curcumin labeling in retinal and brain tissues consistently colocalized with $6 \mathrm{E} 10 \mathrm{mAb}$ labeling and with other $\mathrm{A} \beta$ epitope-specific mAbs, such as $\mathrm{A} \beta_{40} \mathrm{C}^{\prime}$-terminus (Supplemental Figure 1B). Intracellular A $\beta$ immunoreactivity was also detected with $12 \mathrm{~F} 4$ and $6 \mathrm{E} 10$ 
A
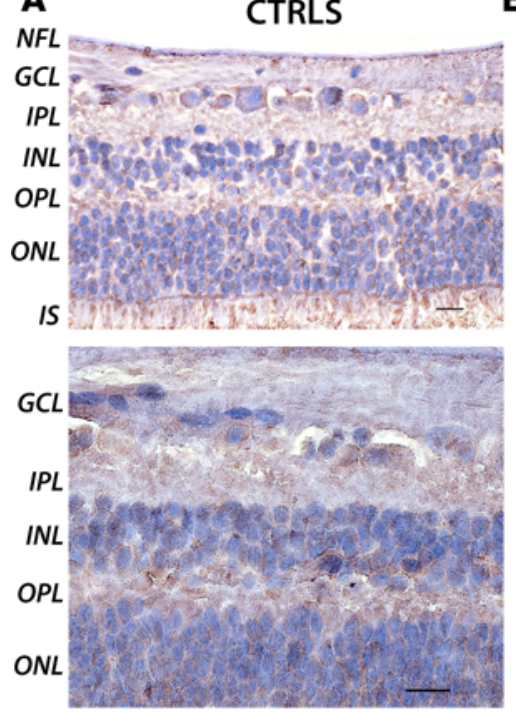

12F4-DAB + Hematoxylin

E
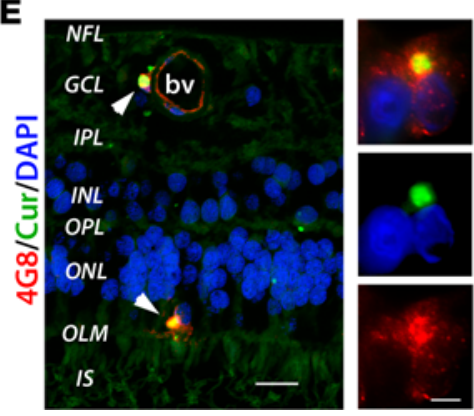

B
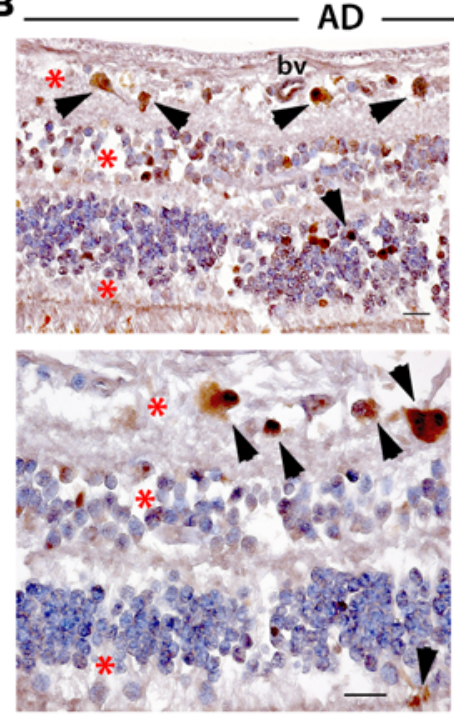

$F$
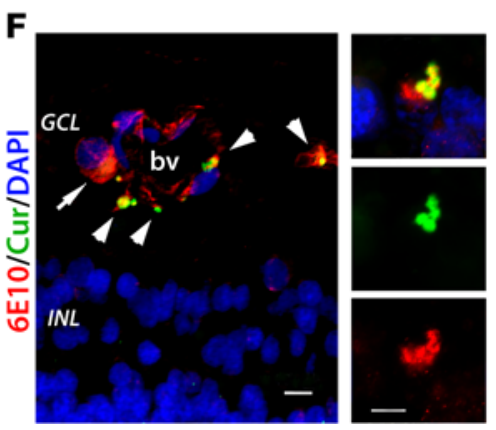

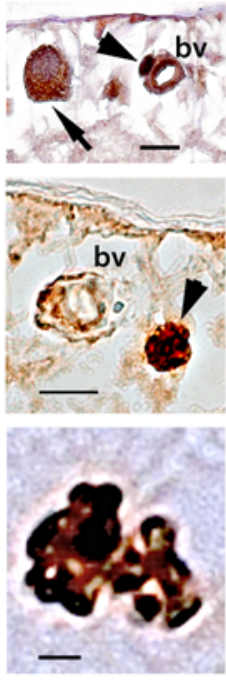

C
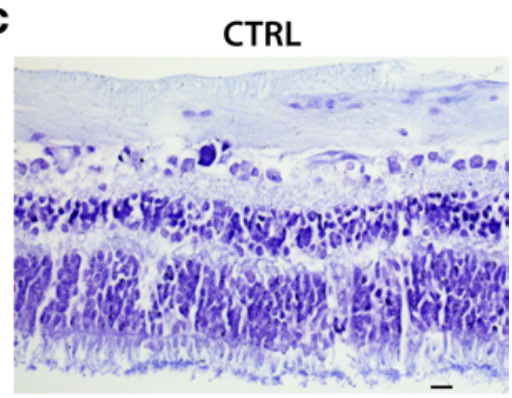

AD
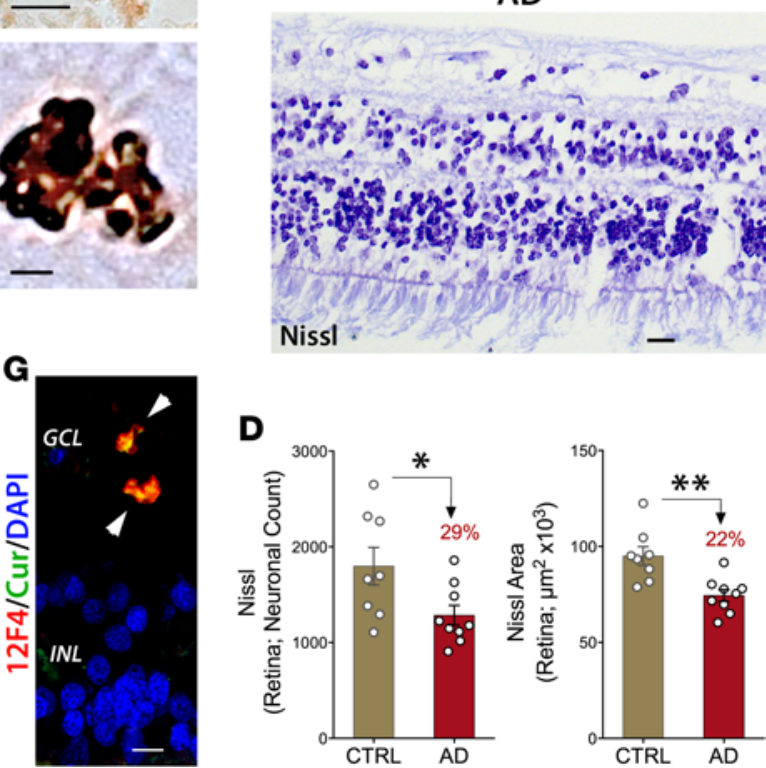

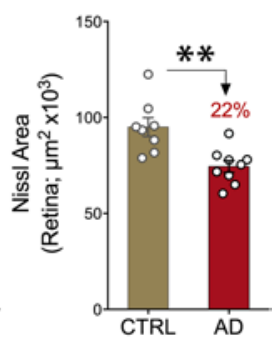

Figure 4. A $\beta$ deposits associated with neuronal loss are detected in the retinas of $A D$ patients. (A and $B)$ Paraffin-embedded retinal cross sections from superior quadrants of AD patients $(n=12)$ and matched CTRLs $(n=8)$ stained with anti-A $\beta 42$ mAbs (12F4) and peroxidase-based labeling (brown). Hematoxylin counter stain for nuclei (violet). (A) Retinas of controls were clear of A $\beta$ immunoreactivity. (B) Retinas of $A D$ patients contained a multitude of $A \beta$ deposits (arrowheads), especially in the ganglion cell layer (GCL). Marked loss of retinal cells apparent in the GCL, inner nuclear layer (INL), and outer nuclear layer (ONL); areas of nuclei loss are indicated by asterisks. Scale bar: $20 \mu \mathrm{m}$. Higher-magnification images are shown. Intracellular cytoplasmic $A \beta$ (top; arrow). $A \beta$ deposits near and inside blood vessel walls (middle; bv; arrowheads); these vascular and perivascular deposits are frequent in GCL. Scale bar: $10 \mu \mathrm{m}$. A compact multicore A $\beta$ deposit found in GCL (bottom; scale bar: $5 \mu \mathrm{m}$ ). (C) Nissl staining of retinal cross sections from a definite AD patient and matched CTRLs ( $n=17$ subjects; experiment repeated 3 times). Altered Nissl neuronal staining is observed in AD patients; changes in cytoplasmic staining patterns (chromatolysis) that could associate with neuronal loss are observed in retinal GCL, INL, and ONL. Scale bar: $20 \mu \mathrm{m}$. (D) Quantitative Nissl neuronal count and total area in a subset of AD patients $(n=9)$ and age- and sex-matched CTRLs $(n=8)$. Percentage change compared with CTRLs is in red. Group mean and SEM are shown. ${ }^{*} P<0.05,{ }^{* *} P<0.01$, unpaired 2-tailed Student's $t$ test. (E-C) Representative images from $n=8 A D$ patients and $n=8$ matched CTRLs; fluorescent images from AD patient retinas showing curcumin-positive $A \beta$ deposits colocalized with various anti-A $\beta$ mAbs (4G8, 6E10, 12F4), recognizing diverse $N^{\prime}$ - and $C^{\prime}$-terminus epitopes within the $A \beta$ sequence (arrowheads). (E) $A \beta$ deposits detected in the ONL, above the outer limiting membrane (OLM), and in the GCL near and inside blood vessel walls (left, arrowheads; scale bar: $20 \mu \mathrm{m})$. Colocalization of curcumin and 4G8 in a retinal A $\beta$ plaque near DAPI nuclear staining demonstrates each unique staining pattern (right; scale bar: $5 \mu \mathrm{m})$. (F) Intracellular/ somatic $A \beta$ immunoreactivity (arrow) and colocalization of 6 E10 with curcumin (arrowheads; left; scale bar: $10 \mu \mathrm{m}$ ). Compact multicore $A \beta$ deposits (right; scale bar: $5 \mu \mathrm{m}$. (C) Curcumin-positive A $\beta$ deposits colocalized with the anti-A $\beta$ mAbs (12F4) in the GCL/IPL (arrowheads). Scale bar: $10 \mu \mathrm{m}$.

staining (Supplemental Figure 1, C and D). Figure 2E shows clusters of A $\beta$ deposits, detected by curcumin and 6E10 fluorescent labeling, resembling those detected by peroxidase-based immunolabeling. Scanning large regions in human retinal flat mounts for $\mathrm{A} \beta$ deposits (stained with $12 \mathrm{~F} 4 \mathrm{mAb}$ ) revealed variability in geometric distribution of amyloid burden (Figure $2 \mathrm{~F}$ ). Our data suggest that $\mathrm{A} \beta$ plaques frequently appear in the periphery of the superior quadrant but are scarce in the posterior pole of the AD patient retina (Figure $2 \mathrm{~F}$ ). Quantitative 12F4-immunoreactive areas of $\mathrm{A} \beta_{42}$-containing plaques (Figure $2 \mathrm{~F}$, geometric regions superior temporal 1-3) in a subset of definite $\mathrm{AD}$ patients versus age- and sex-matched controls indicated a significant 4.7-fold increase in retinal A $\beta$ deposits in AD patients (Figure 2G; $P=0.0063$, Student's $t$ test).

Congo red staining in retinal tissues in a subset of patients and controls revealed extensive Congo redpositive plaque along blood vessels (perivascular) in $\mathrm{AD}$ patients (Figure $3 \mathrm{~A}$ ), whereas the control retina 
was negative (data not shown). The birefringence of Congo red staining under polarized light indicated the presence of amyloid fibrils (Figure 3A).

Retinal $A \beta$ and neuritic-like deposits, NFT-like structures, and fibrillar $A \beta_{42}$ ultrastructures in the innermost layers. Radial cross sections, extending from the ora serrata to the optic disc (Figure 1C), were obtained from retinal superior temporal and inferior-nasal quadrants. Gallyas silver staining, a standard technique for detecting cerebral senile plaques and NFTs, was utilized to further demonstrate the presence of AD-associated neuropathologies in the retinas of $\mathrm{AD}$ patients versus controls (Figure 3, B-E, and Supplemental Figure 3). Cross sections from matched controls exhibited intact retinal tissue lacking major protein aggregates (Figure $3 \mathrm{~B}$ ). In contrast, retinas of definite $\mathrm{AD}$ patients contained multiple extracellular $\mathrm{A} \beta$ deposits (Figure 3, $\mathrm{C}$ and D), including classical plaques (Figure $3 \mathrm{C}$, middle) and deposits associated with blood vessels, especially in the ganglion cell layer (GCL; Figure 3, C and D). Importantly, we identified classical neuritic (senile-like) plaques in the RGC layers (Figure 3C, right, higher-magnification image below). Intracellular-cytoplasmic protein aggregates were also observed (Figure 3E). Furthermore, some neurons in the innermost retinal layers of AD patients exhibited tangle-like morphology resembling NFTs (Supplemental Figure 3).

Next, we immunolabeled retinal cross sections with various anti-A $\beta$ mAbs and hematoxylin nuclear counterstain (Figure 4). While control retinas were typically clear of $A \beta$ immunoreactivity (Figure 4A), definite $\mathrm{AD}$ patients displayed diverse forms of 12F4-positive $\mathrm{A} \beta$ deposits, which often associated with blood vessels, especially in the GCL (Figure 4B and Supplemental Figure 1, E-G). Cytoplasmic A $\beta$ was also detected (Figure 4B, top, and Supplemental Figure 1E), as well as deposits within (Figure 4B, top) or adjacent to blood vessel walls (Figure 4B, right, and Supplemental Figure 1E). Globular plaques consisting of multiple amyloid cores were also observed (Figure 4B, bottom, and Supplemental Figure 1G). Intracellular staining of $A \beta$ was occasionally observed in foam-like pericytes (69) with poorly defined nuclei (Figure $4 \mathrm{~B}$, top, arrowhead). Importantly, along with accumulation of $\mathrm{A} \beta$, a marked loss of retinal cells was apparent in the GCL, inner nuclear layer (INL), and outer nuclear layer (ONL) in AD patients as compared with retinas of matched controls (Figure 4B, red asterisks, vs. Figure 4A). Indeed, altered Nissl neuronal staining was observed in retinal GCL, INL, and ONL of AD patients versus controls, suggesting chromatolysis that may associate with neuronal degeneration (Figure 4C). Quantitative Nissl neuronal count and total area in a subset of AD patients and age- and sex-matched controls $(n=17)$ validated a significant neuronal loss in the retinas of $\mathrm{AD}$ patients (Figure 4D; $P<0.05$ and $P<0.01$, respectively, Student's $t$ test).

Fluorescent imaging demonstrated the colabeling of curcumin with various anti-A $\beta$ mAbs detecting multiple intracellular and extracellular deposits across retinal layers in $\mathrm{AD}$ patients, especially in the GCL but also above the outer limiting membrane (Figure 4, E-G). Staining patterns of curcumin-positive plaques, intensely labeling the central dense $\mathrm{A} \beta$ core (Figure $4, \mathrm{E}$ and $\mathrm{F}$, right), were comparable to those found in retinal flat mounts and brains (Figure 2D). Globular 12F4- and 6E10-positive plaques containing multiple amyloid cores were also detected (Figure 4, F and G).

Transmission electron microscopy (TEM) of vertical and en face (horizontal) retinal sections from AD patients that had been immunoperoxidase labeled with $12 \mathrm{~F} 4 \mathrm{mAbs}\left(\right.$ anti-A $\left.\beta_{42}\right)$ revealed the ultrastructures of retinal $\mathrm{A} \beta$ plaques containing a central dense amyloid core, with and without radiating fibrillar arms, fibrils, protofibrils, and other types of $A \beta$ deposits (Figure 5). Similar to those in the brain, retinal A $\beta$ protofibrils and fibrils were 10 - to 15 -nm wide $(70,71)$ (Figure $5 \mathrm{~A}$, higher magnification) with parallel $\beta$-sheets adjacent to $\mathrm{A} \beta$ deposition (Figure $5 \mathrm{~B}$ ). Ultrastructural examination of the $\mathrm{A} \beta$ plaque site adjacent to a blood vessel basement membrane (Figure 5C, left) revealed structures resembling paranuclei $(72,73)$ that also appeared positive for $A \beta$ - possible $A \beta_{42}$ oligomer assemblies (Figure $5 \mathrm{C}$, arrowhead). TEM images of en face retinal sections further indicated the presence of retinal $\mathrm{A} \beta$ plaques with radial fibril arms emanating from central cores (Figure 5D).

Development of retinal amyloid imaging in live AD patients. Figure 6 illustrates the development of a curcumin regimen and the feasibility of using a noninvasive ophthalmic device to detect increased curcumin fluorescence in retinas of living human subjects. Initially, several commercially available curcumin brands were analyzed for their absorption into the blood and brain, and the ratios of curcumin concentrations in the brain and red blood cells (RBCs) were determined in wild-type mice (Supplemental Figure 4). The highest brain concentrations were obtained with Longvida Optimized Curcumin formulation coated with lipophilic matrix $(74,75)$ (Supplemental Figure 4). The optimal curcumin formulation, dose, and regimen were further calibrated through in vivo retinal amyloid imaging in double-transgenic $\mathrm{APP}_{\mathrm{SWE}} / \mathrm{PS}_{\triangle \mathrm{E} 9}$ mouse models (Supplemental Figure 5). To this end, we tested water-soluble versus solid lipid-based (SLCP) curcumin 
A

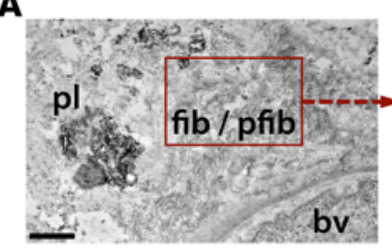

B

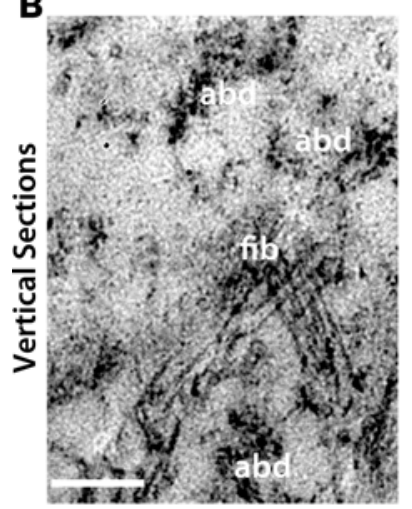

D

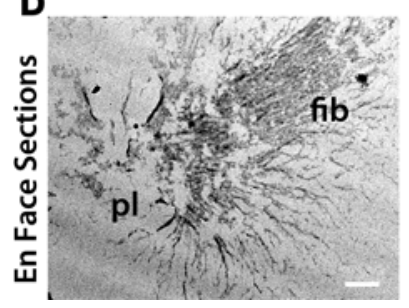

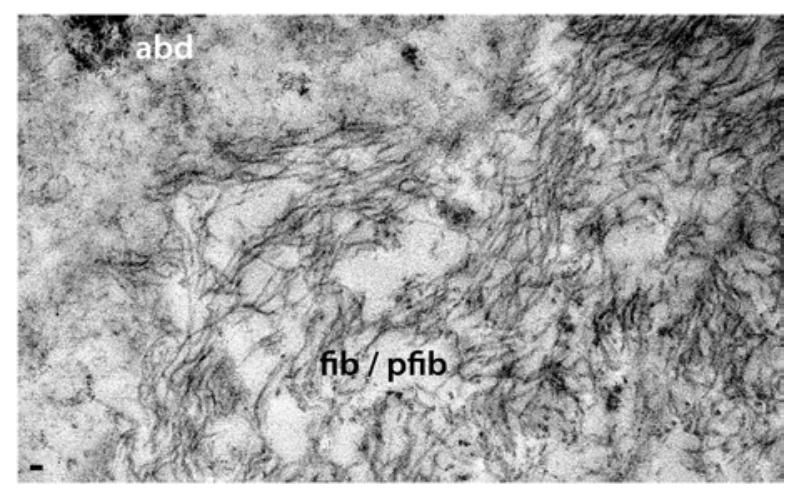

C
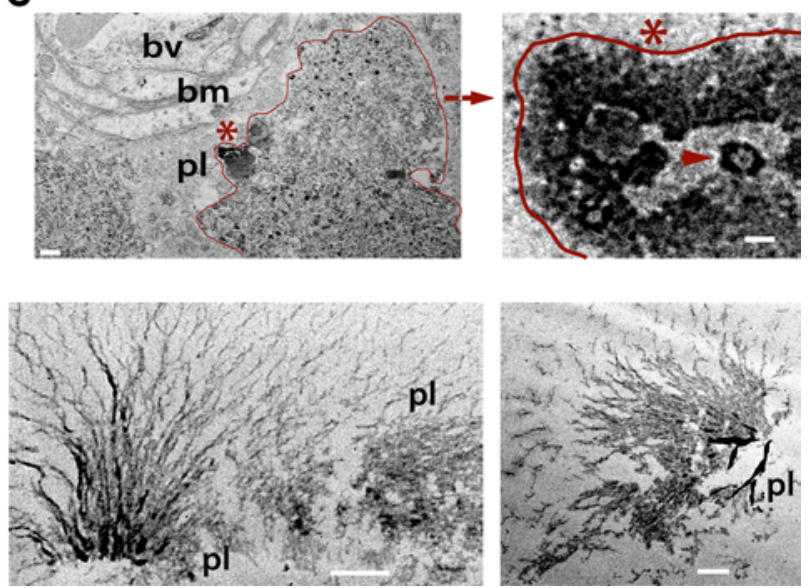

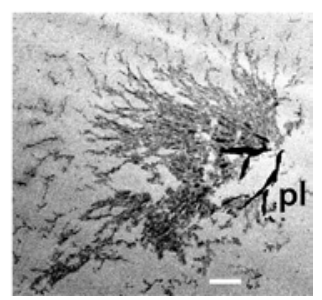

Figure 5. Ultrastructures of $A \beta$ deposits in human AD retina. Transmission electron microscopy (TEM) analyses of vertical (cross; A-C) and en face (horizontal; D) retinal sections from definite AD patients ( $n=3$; experiments repeated 3 times). Retinas were prestained with anti-A $\beta_{42} \mathrm{mAb}(12 \mathrm{~F} 4)$ and a high-sensitivity immunoperoxidase-based system and DAB substrate chromogen. (A) TEM image showing ultrastructure of $A \beta$ plaque (pl), fibrils (fib), and protofibrils (pfib) near a blood vessel (bv) in vertical sections. Scale bar: $1 \mu \mathrm{m}$. Higher-magnification image (right), indicating presence of 10 - to $150-n m$-wide $A \beta$ fibrils as well as protofibrils and $A \beta$ deposits (abd; scale bar: $50 \mathrm{~nm}$ ). (B) Fibrillar $A \beta$ ultrastructure surrounded by multiple $A \beta$ deposits. Scale bar: $200 \mathrm{~nm}$. (C) $A \beta$ plaque-like structure, marked by an asterisk and bordering red line, in close proximity to basement membrane (bm) of a blood vessel (scale bar: $0.5 \mu \mathrm{m}$ ). Higher-magnification image showing $A \beta$ plaque-like area, with structures resembling paranuclei containing annular oligomers (arrowhead; scale bar: $40 \mathrm{~nm}$ ). (D) TEM images of en face sections demonstrating retinal $A \beta$ plaque ultrastructures, with radial fibrillar arms emanating from a central dense core found in the innermost retinal layers. Darker black signal represents condensed $A \beta$ aggregate core. Scale bar: $0.5 \mu \mathrm{m}$.

formulations (Supplemental Figure 5C), given intravenously or orally (Supplemental Figure 5, A and C-F) at varying dosages (Supplemental Figure 5, D-F). Altogether, we found that SLCP Longvida curcumin had better tissue bioavailability, showing maximum signal-to-noise ratio, which led to its selection for the proofof-concept retinal amyloid imaging clinical trial. We next tested a model human eye with various marketed and modified human ophthalmic devices (Supplemental Figure 6, A and B). Alterations to ophthalmic devices included a wide-angle view, multiple-frame capture, and insertion of fluorescent filters. Examples of data exclusion and retinal amyloid imaging in live $\mathrm{AD}$ patients, comparing postacquisition processed images in selected ophthalmic devices, are shown in Supplemental Figure 6, C-G.

Human subjects received oral curcumin for either 2 or 10 days. Their retinas were imaged using a scanning laser ophthalmoscope (SLO) at day 0 (baseline prior to curcumin uptake) and throughout the experiment (Figure 6A). Fundus images from an $\mathrm{AD}$ patient receiving curcumin for 10 days showed increased fluorescent intensity in retinal deposits at days 1 and 10 compared with baseline (Figure 6B). At day 29, a noticeable decay in retinal spot fluorescent intensity was observed (Figure 6B). Spot line profile analysis (Figure 6C) for a particular retinal plaque (Figure 6B) following curcumin uptake indicated increased fluorescent intensity signal at days 1 and 10 and a decay to baseline level at washout (day 29). Representative fundus images from another patient on a 10-day oral curcumin protocol indicated the existence of retinal curcumin-amyloid deposit clusters, seen at days 2 and 10 versus baseline (Figure 6D). After 10 days of curcumin uptake, marked increases in curcumin fluorescence, deposit number (119 spots), and area (1,269 $\mu \mathrm{m}^{2}$ ) were detected compared with baseline (12 spots and $38 \mu \mathrm{m}^{2}$, respectively; Figure $6 \mathrm{D}$, bottom). Pharmacokinetic analysis in six healthy individuals on a 10-day regimen of oral curcumin indicated a significant increase in curcumin levels in both free plasma and RBCs (reflecting tissue absorption), especially from day 3 onward (Figure 6E). Curcumin absorption peaked significantly at day 10 and decreased after 30 days (Figure 6E). Reduction of curcumin to its metabolites in vivo in the plasma and RBC of the above healthy individuals and the effect of $\mathrm{pH}$ on curcumin degradation ex vivo in human blood samples were also measured (Supplemental Figure 7). Repeated retinal imaging in a mild AD patient and a healthy control subject on a 2-day oral curcumin regimen indicated increased retinal integrated fluorescent intensities for 
A

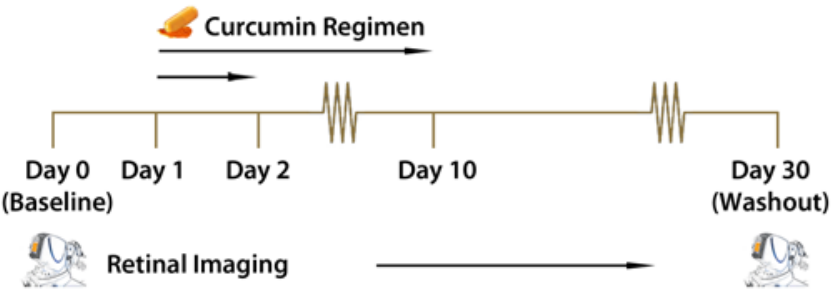

B

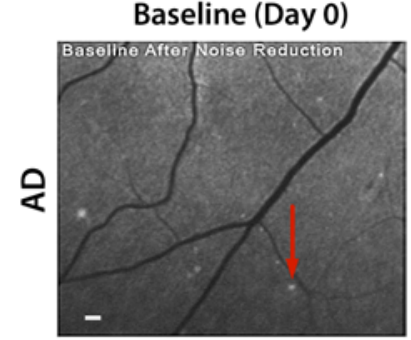

Day 1

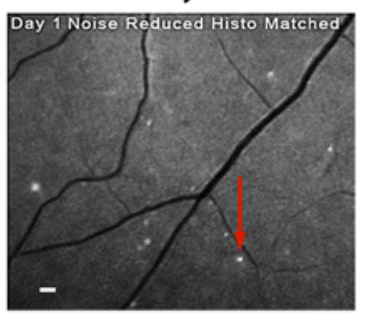

Day 10

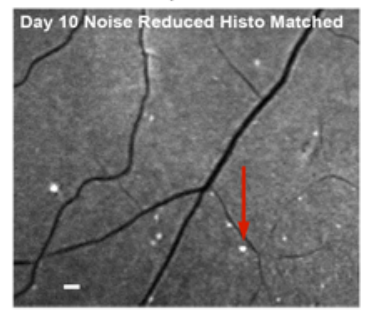

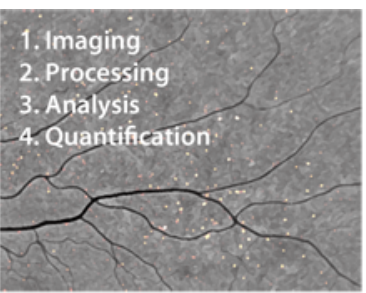

Detection of Curcumin Signal in AD Retina

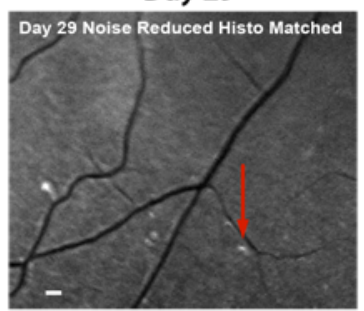

\section{C}
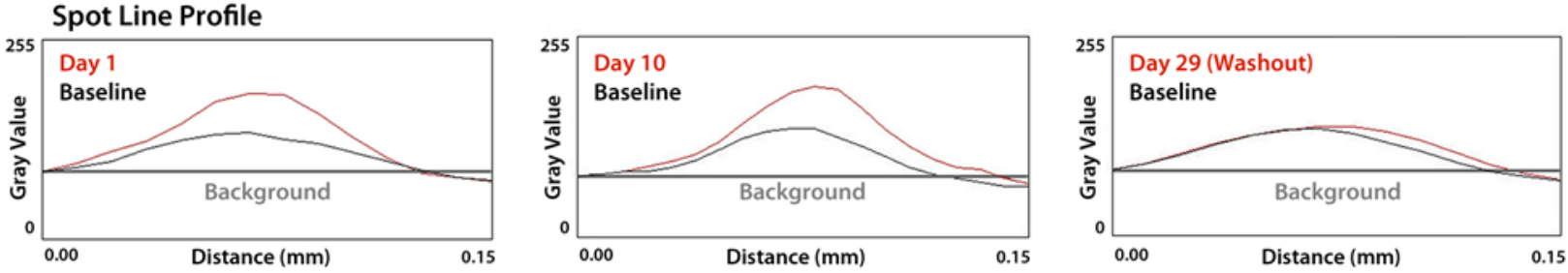

D
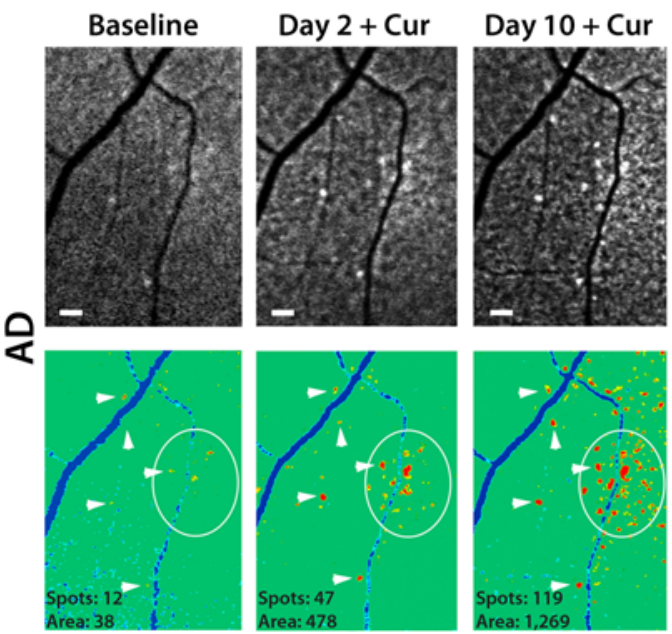

$E$

Blood Analysis

$\mathbf{F}$

Retinal Imaging
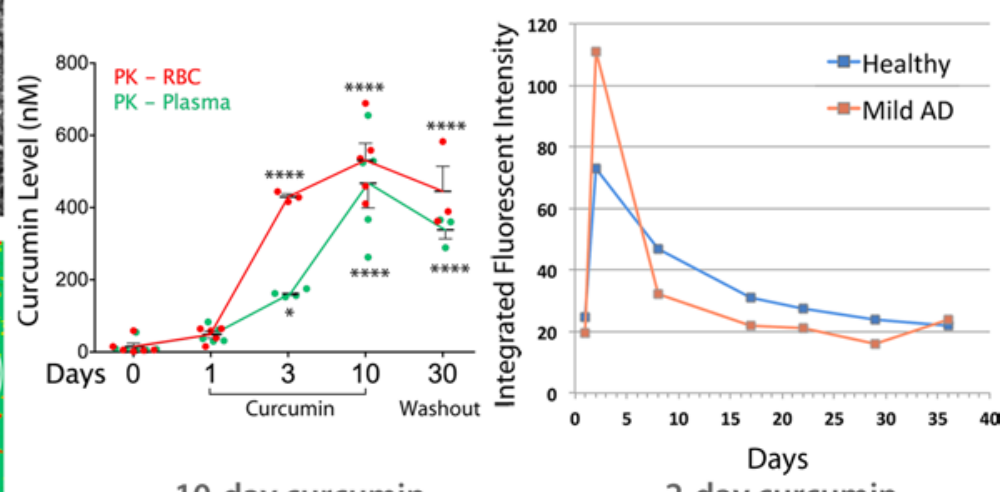

10-day curcumin

Figure 6. Development of a noninvasive retinal amyloid imaging method using curcumin labeling in live human patients. (A) Curcumin regimen and retinal imaging protocols in living human subjects ( $n=16 \mathrm{AD}$ and CTRLs and $n=2 \mathrm{AMD}$ patients). Oral Longvida curcumin administration for 2 or 10 days. Subjects' retinas were imaged with modified ophthalmoscopes prior to (day 0 , baseline image) and after curcumin intake, per the regimen. (B) Repeated regional fundus imaging in a mild $A D$ patient receiving curcumin for 10 days. Increase of curcumin fluorescent intensity due to retinal amyloid deposits (white spots) is observed from baseline (day 0) through days 1-10. Decreased curcumin signal is observed at day 29 (washout). (C) Spot line profile analysis of curcumin fluorescence from an individual plaque (marked in B by red arrows), showing increased signal at days 1 and 10 versus baseline levels. At day 29, fluorescent signal decays to baseline levels (washout). Representative spot line profile from $n=3$ patients. (D) Representative retinal fundus images from a moderate $A D$ patient (top) and their pseudocolor images (bottom); arrowheads mark individual plaques, and circles demarcate a cluster of deposits. Increased spot number and area from baseline image to day 2 and 10 images were quantified by Imagej analysis (bottom). (B-D) Representative images from all human subjects ( $n=16$; Supplemental Table 2). (E) Longvida curcumin pharmacokinetic (PK) analyses in living healthy controls receiving curcumin for 10 days ( $n=6$ subjects; all or a subset analyzed for each time point, as shown). Tissue curcumin levels in red blood cells (RBCs) and free curcumin in plasma peaked at day $10(P<0.0001)$. Group mean and SEM are shown. ${ }^{*} P<0.05$, ${ }^{* * *} P<0.0001$ for comparison between day 0 and days 3 , 10 , or 30 , by 1 -way ANOVA and Tukey's post test. Logarithmic transformation analysis of covariance (subject $\times$ day) demonstrated that curcumin levels increased with duration of treatment in RBCs $\left(R^{2}=0.654\right.$; day: $P=0.001$; subject: $\left.P=0.024\right)$ and plasma $\left(R^{2}=0.559\right.$; day: $P=0.003 ;$ subject: $\left.P=0.09\right)$. (F) A quantitative longitudinal retinal curcumin imaging in a representative healthy control and mild AD patient, both receiving 2-day curcumin regimen. Exponential decay of integrated fluorescent intensity occurred after day 2 . Decay rate $=10.4 \%$ per day, half-life $=6.3$ days, offset IFI $=21.5$. Scale bar: $200 \mu \mathrm{m}$. 


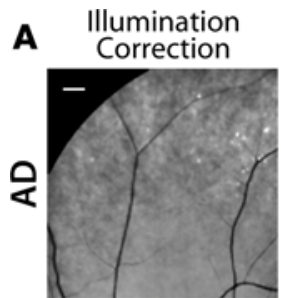

B

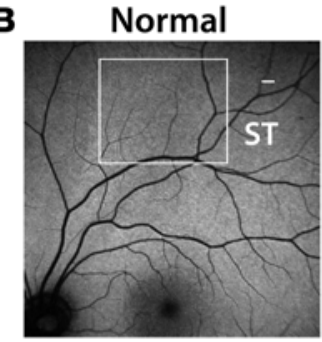

C
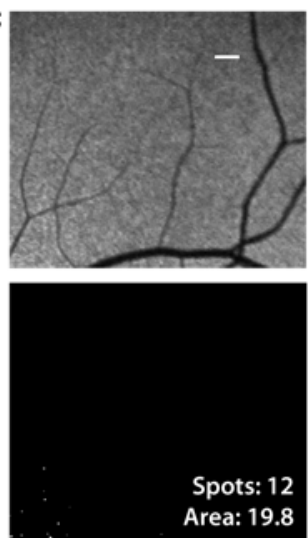

D

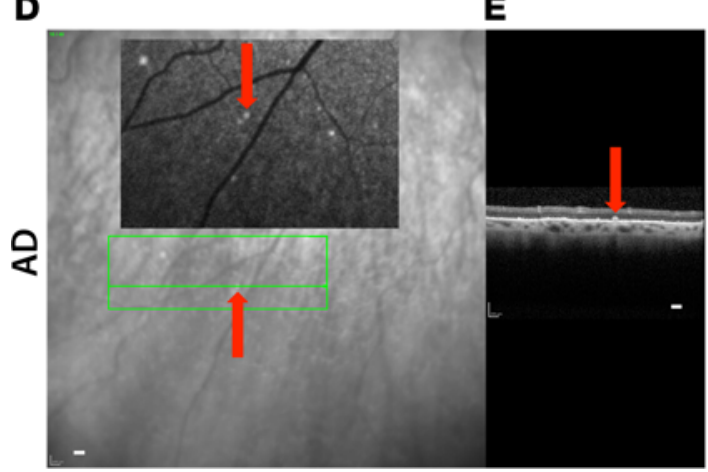

$A D$
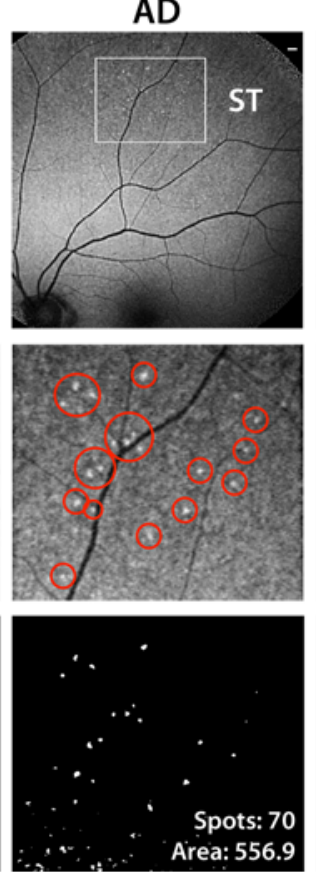
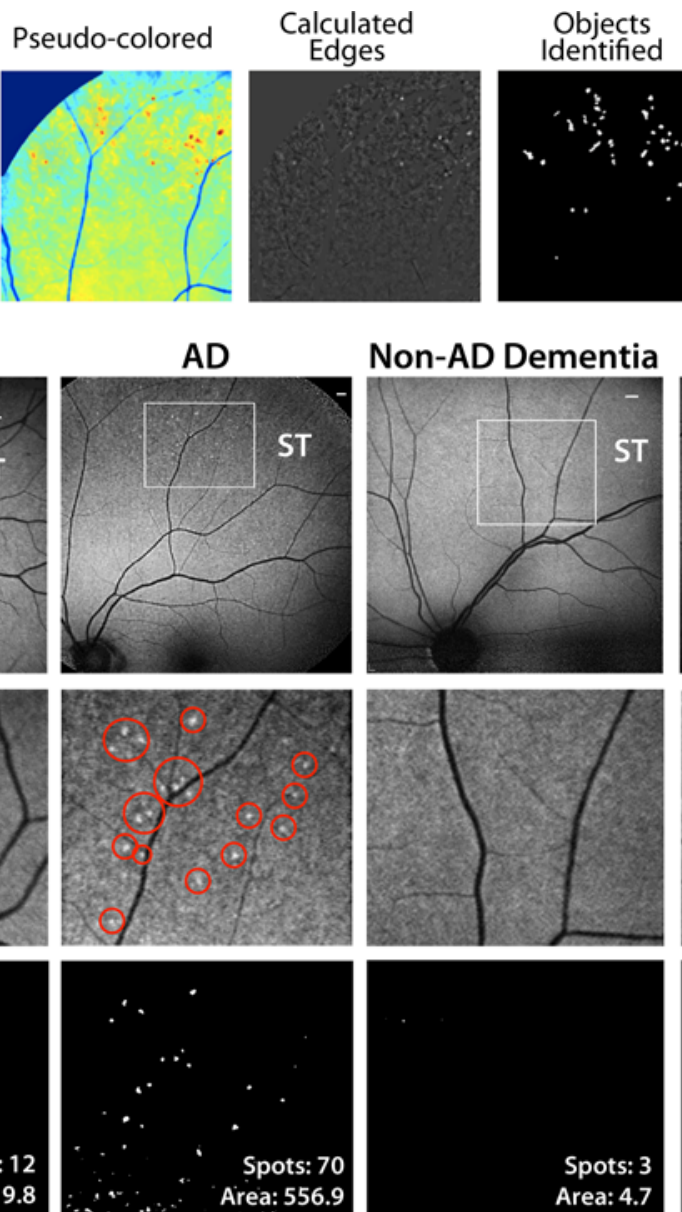

$\mathbf{F}$

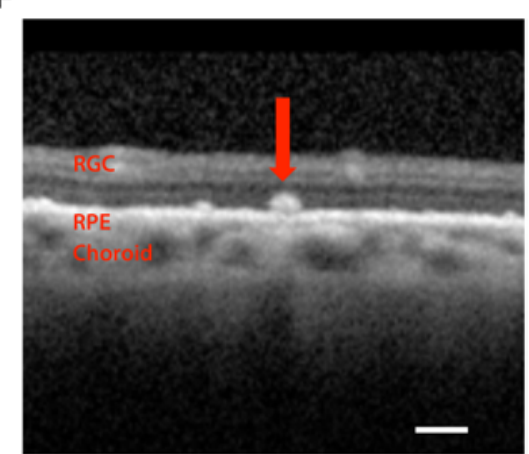

Figure 7. Noninvasive detection of retinal amyloid deposits in live AD patients. (A) Multistep manual postacquisition image processing and analysis to detect and quantify spots with increased curcumin fluorescence signal in the retina (superior quadrant; representative images of $n=16$ human subjects). Scale bar: $800 \mu \mathrm{m}$. (B) Representative images demonstrate detection of retinal curcumin spots in two live $A D$ patients and contrasting minimal spots in a healthy control (Normal) and patient suffering from vascular dementia (Non-AD Dementia). Curcumin fluorescence fundography of superior temporal region (ST) reveals amyloid deposits often in retinal peripheries. Amyloid deposit detection in inferior temporal (IT) region of another AD patient. Regions of interest are indicated by white squares. Scale bar: $400 \mu \mathrm{m}$. (C) Higher-magnification image of the above regions of interest. Red circles in the top images highlight retinal spots of curcumin-increased fluorescence. Scale bar: $400 \mu \mathrm{m}$. Bottom images display postprocessing spot number and fluorescent area $\left(\mu \mathrm{m}^{2}\right)$. ( $\mathbf{D}$ and E) Representative optical coherence tomography (OCT) of a selected curcumin-positive region in an $A D$ patient with no maculopathy (repeated experiments in $n=3$ patients). Scale bar: $200 \mu \mathrm{m}$. (D) Curcumin fluorescence fundography indicating certain retinal amyloid plaque with red arrows. Green lines delineate region of OCT segmentation. (E) Retinal cross section by OCT reveals amyloid plaque in outer retinal layers. (F) High-magnification OCT image displays curcumin-positive deposit located above retinal pigment epithelium (RPE), along with intact RPE and Bruch's membrane.

both $\mathrm{AD}$ and healthy subjects (Figure $6 \mathrm{~F}$ ). Peaks of increased curcumin fluorescence were assessed. The highest integrated intensity levels were measured at day 2 , showing a higher peak in the AD patient, and their exponential decays to basal levels (washout) were observed up to day 36 (Figure 6F).

Fundus images from live patients underwent semiautomated postacquisition processing to optimize detection of curcumin-positive retinal amyloid spots (Figure 7A). The ability to detect individual retinal deposits allowed calculation of their number and area in certain regions of interest (in frame) and comparison among a healthy control, $\mathrm{AD}$ patients, and a non-AD dementia patient (Figure 7, B and C). Representative images show the superior temporal and inferior temporal quadrants (Figure 7B). The retinas of the two representative $\mathrm{AD}$ patients exhibited higher deposit number and area in the superior temporal and inferior temporal quadrants compared with a healthy control (Figure 7C). The patient with non-AD dementia (vascular dementia) had almost no positive spots (Figure 7, B and C). To examine retinal layer location in vivo for a subset of deposits, we utilized optical coherence tomography (OCT; Figure 7, D-F). A representative 
Table 1. Retinal amyloid index and retinal curcumin-positive deposit number in living AD patients and healthy controls

\begin{tabular}{|c|c|c|c|c|}
\hline & \multicolumn{2}{|c|}{ CTRLs } & \multicolumn{2}{|c|}{ AD patients } \\
\hline & RAI & Spot no. & RAI & Spot no. \\
\hline Mean & $30.2 \pm 5.2$ & $24.8 \pm 6.2$ & $78.9 \pm 7.2^{A}$ & $50.2 \pm 7.3^{B}$ \\
\hline Minimum & 18.4 & 2 & 43.9 & 17 \\
\hline Maximum & 46.3 & 43 & 112.4 & 82 \\
\hline Coefficient of variation & $42.0 \%$ & $61.4 \%$ & $29.0 \%$ & $45.8 \%$ \\
\hline MMSE & \multicolumn{2}{|c|}{$N / A^{c}$} & \multicolumn{2}{|c|}{$19.5 \pm 1.2$} \\
\hline Age (yr) (n) & \multicolumn{2}{|c|}{$53.0 \pm 7.7(n=6)$} & \multicolumn{2}{|c|}{$76.0 \pm 2.7^{\mathrm{B}}(n=10)$} \\
\hline Sex & \multicolumn{2}{|c|}{$4 \mathrm{~F}, 2 \mathrm{M}$} & \multicolumn{2}{|c|}{$7 \mathrm{~F}, 3 \mathrm{M}$} \\
\hline Age subgroup (n) & \multicolumn{2}{|c|}{$59.2 \pm 5.4(n=5)$} & \multicolumn{2}{|c|}{$69.8 \pm 1.7^{0}(n=6)$} \\
\hline \multicolumn{5}{|c|}{$\begin{array}{l}\text { Retinal amyloid index (RAI) scores and number of curcumin-positive deposits (spot no.) in } 16 \text { human subjects across a wide age range with clinical AD } \\
\text { diagnosis or healthy controls (CTRLs; extended details on human subjects in Supplemental Table } 2 \text { ). }{ }^{A} P<0.0005,{ }^{B} P<0.05,2 \text {-tailed Student's } t \text { test in } \\
\text { comparison between AD patients and CTRLs. 'N/A, Mini Mental State Examination (MMSE) is not available for asymptomatic CTRLs. These individuals } \\
\text { show no evidence of memory or cognitive executive impairment according to self-reports and/or family members, nor any deficit in activity of daily living. } \\
\text { Data presented for } 10 \text { AD patients and } 6 \text { CTRLs. Age subgroup constitutes } 6 \text { AD and } 5 \text { CTRL subjects with no statistical difference }{ }^{D} \text { in mean age (RAI scores } \\
\text { presented in Figure } 8 \mathrm{I} \text {, right). } n \text {, sample size. F, Female; M, Male. Data presented as mean } \pm \text { SEM. }\end{array}$} \\
\hline
\end{tabular}

amyloid deposit (Figure 7, D-F, red arrows) within a region showing increased curcumin fluorescence in fundus images of an $\mathrm{AD}$ patient was selected for focal scanning by OCT. A higher-magnification OCT image demonstrates an intact retinal pigment epithelium (RPE) and Bruch's membrane and indicates the location of this curcumin-amyloid deposit above the RPE, at the outer retinal layers (Figure 7F).

Increased retinal amyloid index scores in AD patients. A proof-of-concept clinical trial was conducted on 16 subjects, including $10 \mathrm{AD}$ patients and 6 healthy controls (Supplemental Table 2). To determine the feasibility of detecting and quantifying retinal amyloid deposits in live human subjects, we selected a group of mild-to-moderate AD patients (showing clear clinical symptoms and expected to be amyloid positive) and compared them to a group of younger, cognitively normal individuals (expected to have low or no retinal amyloid burden). Although these groups show a significant difference in age, we selected a subgroup of subjects whose age is not significantly different (see Table 1 and Supplemental Table 2) to compare retinal amyloid burden.

Representative images from the fully automated and blinded image-processing steps, involving conversion of raw retinal images and calculation of retinal amyloid index (RAI) scores, are shown in Figure 8, A-G. Retinal Z-stack images were obtained using a SLO. These fluorescent fundus images underwent a controlled and uniform image-processing analysis to improve signal, reduce noise, and identify spots with significantly increased fluorescence (Figure 8, A-C). A spot identification threshold was set for the processed images (Figure 8D). To determine RAI scores, only deposits with increased fluorescent intensity compared with baseline were included in our calculations (Figure 8E, deposits denoted as red dots above the threshold green line). The integrated intensity values for an AD patient with an RAI score of 55.7 and 65 positive deposits are displayed in contrast to a healthy control with an RAI score of 11.2 and 15 positive deposits (Figure 8E, top vs. bottom, respectively). In AD patients, most amyloid deposits were found as clusters in the peripheral superior quadrants, often along blood vessels (Figure 8F), in agreement with histological data (Figures 2-5). Heatmaps and overlay images were created to illustrate overall location and distribution of positive amyloid signal in the context of fine retinal structures (Figure 8G). In addition, two cognitively normal (non-AD) patients with dry age-related macular degeneration (AMD) underwent the same retinal curcumin imaging protocol; their images showed increased curcumin signal in large diffuse lesions at the posterior pole, around and inside the macula (Supplemental Figure 8).

Analysis of RAI scores and spot numbers for human subjects included in the trial are summarized in Table 1 and Supplemental Table 2. There was a significant $(P=0.0005$, Pearson's $r$ test $)$ linear correlation between RAI scores and retinal deposit number (Figure $8 \mathrm{H})$. AD patients showed increased RAI scores and spot numbers as compared with the younger healthy controls (Figure 8I, left; $P=0.0003$, Student's $t$ test). In accordance with RAI scores, the number of retinal deposits (spots) was elevated in AD patients (Supplemental Table 2). In a subgroup of AD patients and healthy controls not differing significantly in mean age ( $n=11$; Table 1$)$, AD patients showed a significant 2.1-fold increase in RAI scores (Figure 8I, 
A

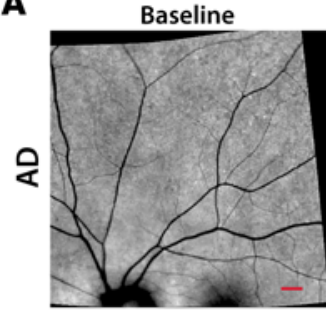

Illumination Correction \& Averaging

Day 2

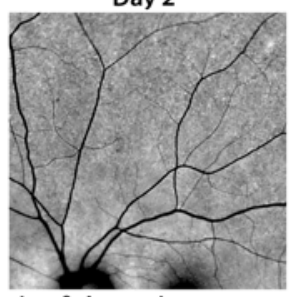

Day 2

C

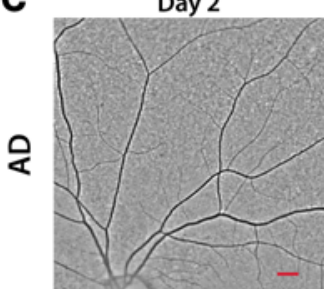

Conversion to MultiScale Gradient Mode

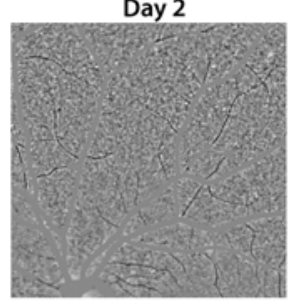

Filtering \& Vessel Masking

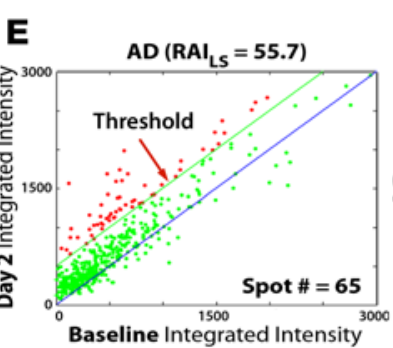

$\mathbf{F}$

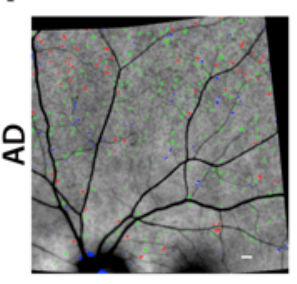

G

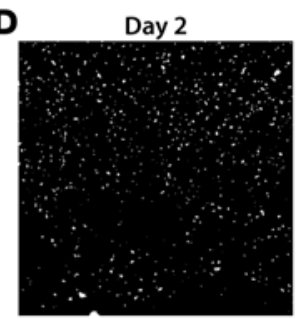

Spot Identification \& Threshold
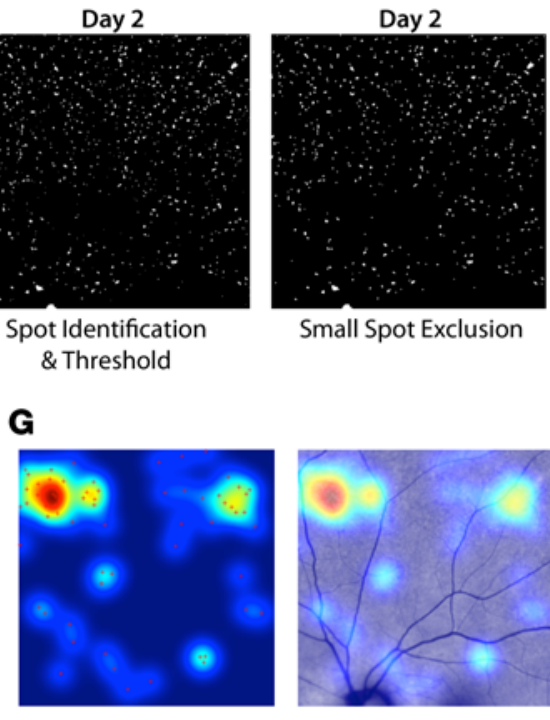

Small Spot Exclusion

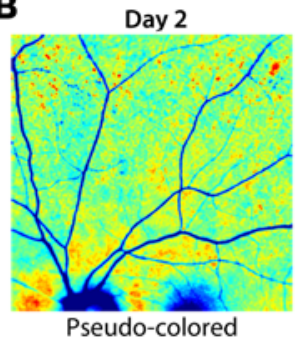

H

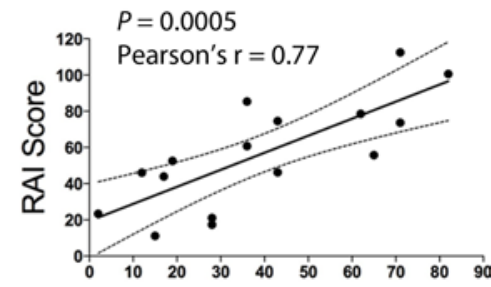

Retinal Spot \#

I
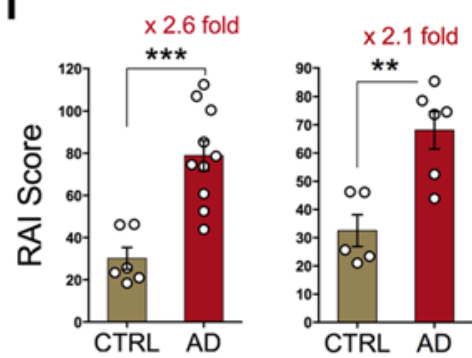

J
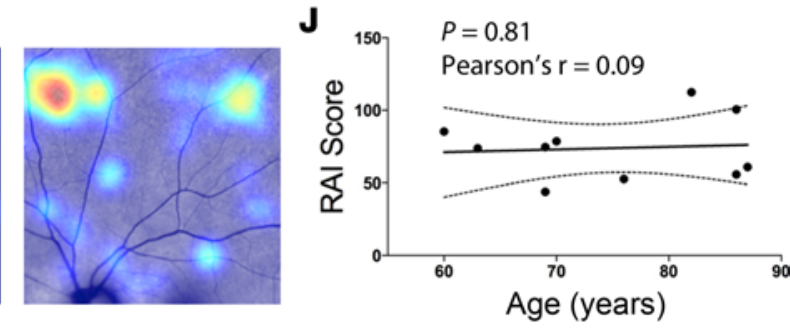

$\mathbf{K}$

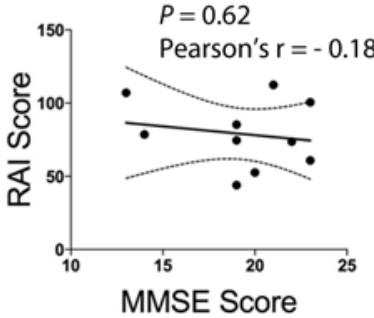

Figure 8. Increased retinal amyloid index in AD patients - a proof-of-concept human trial. (A-D) Representative automated image-processing sequence after repeated fundus image acquisition at day 0 (Baseline) and day 2 , showing retinal superior temporal region in an $A D$ patient. (A) Collection of $Z$-stack scanning laser ophthalmoscope (SLO) fundus images. Each, at baseline and on day 2, underwent illumination correction, alignment of baseline to day 2, and contrast enhancement. (B) Pseudocolor image for visualization. (C and D) Multistep automated postacquisition image processing. (E-G) Threshold defining increased curcumin fluorescent signal in left eye superior hemisphere (LS) and calculation of retinal amyloid index (RAI) scores in a mild AD patient and age-matched CTRL. (E) Blue lines are 1:1 reference, and green lines represent the threshold level, determined at 500 counts and above (red spots are above the threshold). (F) Color-coded spot overlay images. Red spots are above threshold and considered curcumin-positive amyloid deposits, green spots exceed 1:1 reference but not threshold, and blue spots fall below reference. (G) Heatmap images with red spot centroids (left). Day 2 combined overlay images show heatmaps with regions of interest (right). The same automated image processing and analysis was applied on all human subjects. (H) Significant Pearson's $r$ correlation between RAI scores and retinal spot number in all data set ( $n=16$ living subjects, see Supplemental Table 2 ). (I) RAI scores in comparison between AD patients $(n=10)$ and non-age-matched CTRLs $(n=6)$ (left) and a comparison of RAI scores in an age-matched subgroup of AD patients ( $n=6)$ and CTRLs ( $n=5$, see Table 1) (right). Group mean and SEM are shown. ${ }^{*} P<0.005$, ${ }^{* *} P<0.0005$, unpaired 2-tailed Student's $t$ test. (J) Pearson's $r$ correlation analysis of RAI scores and age. (K) RAI and MMSE cognitive scores in AD patient group. Scale bar: $800 \mu \mathrm{m}$ (A-D); $400 \mu \mathrm{m}$ (F and G).

right; $P=0.0031$, Student's $t$ test). In our clinical setup, RAI scores were neither correlated with age of AD patients (Figure 8J) nor with their Mini Mental State Examination (MMSE) scores (Figure 8K).

\section{Discussion}

This study demonstrates the feasibility to noninvasively detect and quantify amyloid deposits in the retinas of live human subjects by using a solid lipid curcumin fluorochrome and a modified point SLO. In a proof-of-concept trial, the mean RAI score in AD patients was elevated compared with that of the healthy 
controls. A histological examination of retinas from neuropathologically confirmed AD patients revealed certain layer and geographical distribution of $\mathrm{A} \beta$ deposits, especially in previously disregarded retinal regions. These results expand past identification of retinal $A \beta$ plaques and $A \beta_{42}$ peptides in the retinas of $\mathrm{AD}$ patients $(26,39,40,42)$ and further demonstrate the existence of classical retinal deposits and morphologies relevant to AD neuropathology, including neuritic-like plaques and NFT-like structures. Retinal deposits frequently appeared in clusters and, although smaller, mirrored $\mathrm{A} \beta$ plaque morphology and vascular pathology in the brain. Retinal plaques were also associated with retinal neuronal loss in these patients. Electron microscopy analysis and Congo red staining verified the existence of retinal A $\beta$ fibrils and protofibrils and the potential existence of $\mathrm{A} \beta$ oligomers. Importantly, $\mathrm{AD}$ patients showed a substantial increase in $\mathrm{A} \beta_{42}$-containing deposits as compared with age- and sex-matched healthy controls. Collectively, these findings suggest the utility of noninvasive retinal amyloid plaque imaging as a surrogate biomarker of $\mathrm{AD}$.

Retinal $\mathrm{A} \beta$ deposits in $\mathrm{AD}$ patients were frequently concentrated in the mid- and far-periphery of the superior quadrants, matching the pattern we detected in living patients via retinal curcumin imaging. These findings, together with superior retina neuronal loss may elucidate previous reports of axonal loss and NFL thinning in these patients $(24-26,29,76-78)$, detected mostly in the same retinal regions that amyloid pathology was evident. Similarly, Blanks and colleagues described an extensive loss of neurons, typically in the superior quadrant and the mid- and far-peripheries (16). The significant NFL reduction in the superior quadrant is consistent with recent findings of mRGC loss and $A \beta$ accumulation in the same retinal regions (26). This increase in $A \beta$ pathology in the superior quadrant, along with severe thinning of the NFL and loss of mRGCs, also known as intrinsically photosensitive RGCs containing melanopsin and implicated in regulation of circadian rhythm (26), may help explain sleep disturbances and visual field dysfunctions in $\mathrm{AD}$, even at early stages $(26,32,44,79)$. Furthermore, the existence of $\mathrm{A} \beta$ deposits in regions in which rod cells are abundant could contribute to the impairments in visual motion and low-contrast sensitivity documented in $\mathrm{AD}$ patients $(80,81)$. In addition, the reason for the geographical predilection of $\mathrm{A} \beta$ accumulation and associated pathology in $\mathrm{AD}$ for the superior quadrant is unknown; we postulate that unique tissue properties and structural parameters of this retinal region perhaps deem it more susceptible to $\mathrm{AD}$ pathology. In the brain, $\mathrm{A} \beta$ plaques are also detected earlier and more frequently in certain brain regions; cerebral $\mathrm{A} \beta$ accumulation is a complex multifactorial process caused by increased production/ aggregation or decreased clearance of $\mathrm{A} \beta$ (reviewed in Zuroff et al., ref. 82). It is possible that differences in retinal blood flow, vessel diameter, choroid thickness, tissue permeability, and light stimulation in this retinal region are some of the factors that may adversely influence $A \beta$ aggregation or clearance.

Histological assessment of $A \beta$ pathology in the human retina, including plaques containing $A \beta_{40}$ and $\mathrm{A} \beta_{42}$ isoforms, was achieved by applying certain isolation techniques and tissue preparation and biochemical labeling protocols as well as screening of large retinal areas (Figure 1). A couple of studies that were unable to detect $\mathrm{A} \beta$ in the human $\mathrm{AD}$ retina analyzed horizontal cross sections spanning a narrow strip from the nasal to temporal quadrants $(41,43)$, rather than screening large regions in flat mounts, thus limiting their analysis to regions that are largely spared of amyloid pathology in $\mathrm{AD}$ patients. Therefore, while Ho and colleagues (43) were unable to detect $A \beta$, p-tau, or $\alpha$-synuclein in any ocular tissue of AD or Parkinson's disease (PD) patients, considerably more independent studies in fact reported the presence of $\mathrm{A} \beta$ deposits in the retina and lenses $(11,39,40,42,51,83)$ as well as identified p-tau (41) and $\alpha$-synuclein (84) in the retinas of AD and PD patients, respectively. Future studies should apply suitable techniques and comprehensive examination of flat-mount retinas to study additional pathological hallmarks of $\mathrm{AD}$ in the retina.

Analysis of retinal cross sections derived from the superior quadrants indicated accumulation of $A \beta$ deposits mostly in the innermost retinal layers (GCL, IPL, and INL). Gallyas silver stain evidenced classical plaques, neuritic (senile-like) plaques, and NFT-like structures in the GCL, although their exact identity requires further study employing specific antibodies against pTau and NFT. In addition, neuronal reduction observed in INL, ONL, and especially GCL of AD patients, quantified by Nissl stain, was accompanied by retinal A $\beta$ pathology. Indeed, the abundance of deposits in the GCL may explain the ganglion cell degeneration and abnormal electroretinogram patterns reported in $\operatorname{AD}$ patients $(26,85,86)$ and animal models of $\mathrm{AD}$ $(22,54,57,87)$. The finding of intracellular $\mathrm{A} \beta$ accumulation in $\mathrm{AD}$ retinas, either somatic or perinuclear, suggests an alternate mode of $A \beta$-mediated toxicity. Recent studies have demonstrated the detrimental effects of intracellular $A \beta$, by way of oligomerization into pathological forms within the endoplasmic reticulum (88), as well as the formation of neuritic plaques from degenerating neurons containing intracellular $A \beta$ (89). 
Ultrastructure examination of $\mathrm{A} \beta_{42}$-containing deposits by TEM revealed the existence of retinal $\mathrm{A} \beta_{42}$ fibrils, protofibrils, and other intermediates in the pathway of $\mathrm{A} \beta$ deposition. We detected assemblies with morphology resembling paranuclei containing annular oligomers $(72,73)$, the building blocks of $A \beta$ protofibrils and fibrils. The concurrent existence of various $A \beta$ structures in the retina of the same patient may suggest that $\mathrm{A} \beta$ accumulates in different disease stages. This supports the hypothesis that different types of $\mathrm{A} \beta$ deposits develop independently rather than sequentially from the same plaque type (90). It is likely that multiple factors contribute to the formation of each type of retinal deposit, but the underlying mechanisms of plaque and nonfibrillar oligomer formation in the retina are not yet understood. This uncertainty merits future investigation of the various plaque phenotypes and distribution of each type in the human retina during normal aging and disease progression $(\mathrm{AD})$.

In this study, we frequently found retinal $A \beta$ deposits in association with blood vessels similar to cerebral vascular amyloid pathology (91). As retinal and cerebral microvasculature share many morphological and physiological properties (34), these vascular amyloid findings may explain the well-documented narrowing of venous blood column diameter and reduced blood flow in both the retinas $(19,20,33,76,92)$ and brains (93-95) of AD patients. Recently, a mechanism for such phenomena was proposed in a study using murine models of $\mathrm{AD}$ demonstrating that vascular amyloid could harden blood vessel walls and decrease blood flow (96). Still, larger studies are required to confirm the association between cerebral amyloid angiopathy and retinal vascular amyloid pathology.

To detect amyloid deposits in retinas of living patients, we translated our retinal curcumin optical imaging approach, previously developed in rodent models of $\mathrm{AD}(40,51)$. Using a modified ophthalmic device, we analyzed single, cluster, and large-area deposits with increased curcumin fluorescent intensity in human subjects. Retinal amyloid deposits at baseline (before curcumin intake) generally exhibited faint autofluorescence. Previous studies also report weak autofluorescence near plaques in the brains of ADtg mice that had never received curcumin $(40,97)$. The low-grade autofluorescence of plaques in the absence of curcumin may result from oxidative damage-induced lipid peroxidation production of aldehydes that create autofluorescent Schiff's bases in and near aggregated amyloids (98). In addition, pigmentation (i.e., lipofuscin accumulation) often occurs in the retinas of elderly individuals and can also generate autofluorescent spots $(99,100)$. Therefore, measuring increased fluorescence of curcumin spots allows for specific detection of $A \beta$-containing deposits in vivo. Importantly, we previously confirmed the identity of retinal amyloid plaques that were imaged in vivo with curcumin in ADtg mouse models by subsequent ex vivo immunolabeling, demonstrating specific anti-A $\beta$ labeling in the same plaques (40). The future availability of postmortem retinal tissues from previously in vivo-imaged human subjects will warrant investigation in order to determine the correlation between RAI scores and retinal histopathology of A $\beta$ deposits.

Curcumin has been reported to have high affinity and specificity for the $\beta$-pleated sheets of $A \beta$ and moderately stains NFTs that are positive for PHF-tau. Moreover, curcumin has high affinity to $\mathrm{A} \beta_{42}$, oligomers, and fibrils, which are tightly linked to $\mathrm{AD}$ (62-68). Curcumin intensely stains the central dense amyloid core in $\mathrm{A} \beta$ fibrils and plaques, as was also previously demonstrated in the brain of murine models of $\mathrm{AD}$ (101). Further, it labels the nonfibrillar forms of $A \beta_{40}$ and $A \beta_{42}$ based on its specific binding to the $A \beta$-hexapeptide ${ }^{16} \mathrm{KLVFFA}^{21}$ amino acid sequence domain $(64,102)$. Previous reports showed that curcumin readily crosses the blood-retinal and -brain barriers in murine models and specifically labels A $\beta$ aggregates $(40,51,103)$. We found here that Longvida oral curcumin formulation consistently colabeled $A \beta$ plaques with various sequence-specific $\mathrm{mAbs}$ in postmortem human retinas and brains, including $12 \mathrm{~F} 4$, which recognizes the $C^{\prime}$-terminus $A \beta_{42}$ alloforms and not APP. Curcumin showed a distinct labeling pattern that was similar to the amyloid spots observed in living patients. Curcumin is considered as generally recognized as safe by the FDA based on previous clinical trials $(74,75,104)$, and the Longvida curcumin used in this study caused minimal to no adverse effects. Longvida's safety has been demonstrated in pharmacokinetic studies in humans (105) and extensive toxicity testing in animals (106), and its efficacy has been demonstrated in two clinical trials $(74,75)$. The choice of curcumin as an amyloid contrast agent in living patients was further supported by the experiments presented here (Figure 6 and Supplemental Figures 4-5 and 7), previously in vivo and ex vivo in ADtg mice $(40,51)$, and in a study using OCT imaging in MCI patients (107). The high bioavailability of optimized curcumin was established by increased tissue absorption of free rather than glucuronidated or other curcumin metabolites from the gastrointestinal system to the blood stream.

An OCT examination in a small subset of patients showed increased curcumin fluorescent spots indicative of amyloid deposits in $\mathrm{AD}$ patients, located in peripheral regions above an intact RPE layer 
and Bruch's membrane and in the absence of maculopathy. These findings are distinct from drusen described in AMD patients (108-110). AMD is a common ocular disease described by sub-RPE drusen deposits, Bruch's membrane thickening, and outer retina degeneration of the RPE and photoreceptors within the macula centralis (111). A $\beta$ immunoreactivity was detected in $30 \%-40 \%$ of soft drusen in AMD patients, often occurring in the posterior pole below a disrupted RPE and Bruch's membrane and along with maculopathy (108-110). Our preliminary data on two AMD patients, using the same retinal curcumin imaging protocol, showed deposits with increased curcumin intensity within large diffuse lesions at the posterior pole/central retina, rather than plaques in the peripheral retina, as detected in AD patients. Further, drusen are often seen as hypofluorescent spots (108-110). Yet, this study cannot rule out the existence of pseudodrusen, also known as subretinal drusenoid deposits, in the retinas of $\mathrm{AD}$ patients, although these are mostly located in the macula in AMD and differ from our in vivo and histological observations in the AD retina. Future studies should determine through large sample sizes whether the location of retinal amyloid pathology in AMD and AD is different, which could help with differential diagnosis.

Glaucoma is another common ocular disease that was previously implicated as sharing commonalities with $\mathrm{AD}$ (reviewed in Hart et al., ref. 11). While retinal pathology found in AD has revealed NFL and GCL loss in addition to hallmark $A \beta$ plaque and tauopathy, predominantly in the innermost retinal layers of the peripheral superior quadrant, glaucoma pathology indicated a particular pattern of central retina neuronal loss in the GCL along with optic disc cupping. This and other pathological evidence relating the two diseases remains quite controversial. Thus, further investigation is needed before positing claims of shared abnormalities.

Within our small AD patient group, we did not obtain a correlation between MMSE scores and RAI scores. Although analyses performed on a limited sample size are challenging to interpret, this result is comparable to amyloid burden in the brain. Cerebral amyloid-PET imaging studies predominantly indicate that amyloid burden plateaus early in the course of $\mathrm{AD}$ (112). Here, in a subset of patients, for which retinal and cerebral amyloid burden was available, we found a significant correlation between superior temporal $\mathrm{A} \beta_{42}$ deposits and Gallyas silver- and thioflavin-S-positive plaques in the brain, which was seemingly stronger for the primary visual cortex. The apparent correlation between retinal and brain plaques calls for further study in larger groups of $\mathrm{AD}$ patients to determine their relationship during disease progression as well as to analyze the association with specific brain regions.

While the timing of $\mathrm{A} \beta$ plaque appearance in the retinas of $\mathrm{AD}$ patients is unknown, our previous histological analysis revealed its existence in early-stage cases (e.g., probable and possible AD) (40). In addition, we detected retinal $\mathrm{A} \beta$ plaques in flat mounts from ADtg mice at least 2 months prior to their presence in the respective hippocampi and cortices (40). These data may suggest that $A \beta$ accumulation in the retina is an early event in $\mathrm{AD}$ pathogenesis, yet large longitudinal studies in presymptomatic $\mathrm{AD}$ and MCI patients are required to confirm retinal $\mathrm{A} \beta$ plaque as an early biomarker. Further, the capability of the RAI score to detect $\mathrm{AD}$ with high sensitivity and specificity should be evaluated in follow-up studies with a greater number of cases compared with age- and sex-matched controls. Such studies could provide a better understanding of $\mathrm{AD}$ manifestation in the retina and further validate our methodology by determining whether retinal amyloid burden is a reliable biomarker of $\mathrm{AD}$.

In summary, we present here a quantitative and detailed histological report of retinal $\mathrm{A} \beta$ deposits and the pathological hallmarks of $\mathrm{AD}$, including their distribution and ultrastructure in $\mathrm{AD}$ patients, together with the demonstrated feasibility to noninvasively image and quantify retinal amyloid deposits in living patients. Such retinal amyloid imaging technology, capable of detecting discrete deposits at high resolution in the CNS, may present a sensitive yet inexpensive tool for screening populations at risk for $\mathrm{AD}$, assessing disease progression, and monitoring response to therapy.

\section{Methods}

Extended methods are provided in the Supplemental Methods.

Postmortem eyes and brains. Donor eyes and brains of AD patients and controls $(n=37)$ were obtained from the University of Southern California Alzheimer's Disease Research Center Neuropathology Core (IRB HS-042071). Clinical and neuropathological data are included in Supplemental Table 1.

$A \beta$ immunohistochemistry. Peroxidase- and fluorescent-based immunostaining of brains and retinas was performed. Images were acquired using a Carl Zeiss Axio Imager Z1 microscope. 
A $\beta$ plaque distribution analysis. The geographical location and layer distribution were determined in flat mounts and cross sections, respectively.

Retinal $A \beta_{42}$ plaque quantification. A $\beta$ plaques were analyzed from retinal superior temporal quadrants.

Brain plaque quantification. A $\beta$ plaques were examined and quantified from 7 brain regions.

Curcumin and Sudan black B staining. Tissues were stained as previously described (40).

TEM. Sections were analyzed on a JEOL JEM-2100 LaB6 TEM and imaged on a Gatan SC1000 Orius.

Curcumin bioavailability in mice. Absorption of curcumin formulations into the blood and brain was measured by HPLC.

Optimization of oral curcumin formulation and regimen for retinal A $\beta$ imaging in live ADtg mice. The procedure was optimized through in vivo retinal imaging.

Curcumin administration in human subjects. A dose of $4 \mathrm{~g}$ Longvida containing $1 \mathrm{~g}$ curcumin was taken once a day for several days prior to imaging.

Curcumin pharmacokinetics in humans. Plasma and RBC samples were analyzed by LCMSMS as previously described (113).

Phantom eye model and ophthalmic device development. A model eye was developed to test device modifications.

Noninvasive retinal amyloid imaging in live human subjects. AD patients and controls $(n=16)$ were imaged; see details on human subjects in Supplemental Table 2.

Imaging protocol. Images were acquired and processed consistently according to a defined procedure.

Semiautomated and fully automated image analysis. Image acquisition and processing protocol was automated for quantification of retinal amyloid burden.

$R A I$ score. RAI score encompasses a number of factors characterizing detected spot number, area, intensity, and distribution.

OCT. OCT scans were performed with Heidelberg Spectralis.

Statistics. GraphPad Prism Software version 6.01 was used for analysis. A comparison of three or more groups was performed using 1-way ANOVA followed by a Bonferroni's or Tukey's multiple comparison post test. Logarithmic transformation analysis of covariance was also applied for pharmacokinetics data analysis. Two-group comparisons were analyzed using a 2-tailed unpaired Student's $t$ test. The statistical association between two or more variables was determined by Pearson's correlation coefficient $(r)$ test. Pearson's $r$ indicates direction and strength of the linear relationship between two variables. Results are expressed as mean \pm SEM. $P$ values of less than 0.05 are considered significant.

Study approval. Clinical trials were approved by Quorum Review, Seattle, Washington, USA (IRB00003226), and the US Department of Health and Human Services (FWA00019841). Human tissues are from the USC ADRC Neuropathology Core, USC, Los Angeles, CA (IRB: HS-042071). Animal studies were approved by the Cedars-Sinai Medical Center Institutional Animal Care and Use Committee and the Division on Laboratory Animal Medicine at the University of California, Los Angeles.

\section{Author contributions}

$\mathrm{MKH}, \mathrm{YK}, \mathrm{SRV}$, and KLB conceived, designed, and supervised the study. YK, DB, SRV, and MKH contributed major experimentation and data acquisition and analysis. EB, DSB, JAP, WJA, SJK, AB, DTF, $\mathrm{AA}, \mathrm{SF}, \mathrm{GMC}, \mathrm{CAM}$, and DRH contributed to experimentation, patient recruitment, and image acquisition. MKH, YK, DB, SF, DRH, SRV, and KLB provided data analysis, interpretation, and presentation. SF and GMC conducted pharmacokinetics experiments and data analysis. MKH, YK, DB, GMC, SF, CAM, DRH, SRV, and KLB discussed intellectual content, supervised, and edited the manuscript. MKH and YK wrote, revised, and provided final approval of the manuscript.

\section{Acknowledgments}

We thank Mia Oviatt, Hannah Schubloom, and Nadav J. Hart for assisting with editing of the manuscript. This paper is dedicated to the memory of Salomon Moni Hamaoui and Lillian Jones Black, both of whom died from Alzheimer's disease. We received support from a National Institute on Aging award (AG044897, Koronyo-Hamaoui, PI) and The Saban and The Marciano Family Foundations (Koronyo-Hamaoui, PI).

Address correspondence to: Maya Koronyo-Hamaoui, Cedars-Sinai Medical Center, 127 S. San Vicente Boulevard, Los Angeles, California, 90048, USA. Phone: 310.423.7473; Email: maya.koronyo@cshs.org. 
1. Alzheimer's Association. 2016 Alzheimer's disease facts and figures. Alzheimers Dement. 2016;12 (4):459-509.

2. De Strooper B, Karran E. The cellular phase of Alzheimer's disease. Cell. 2016;164(4):603-615.

3. Hardy J, Duff K, Hardy KG, Perez-Tur J, Hutton M. Genetic dissection of Alzheimer's disease and related dementias: amyloid and its relationship to tau. Nat Neurosci. 1998;1 (5):355-358.

4. Bateman RJ, et al. Clinical and biomarker changes in dominantly inherited Alzheimer's disease. N Engl J Med. 2012;367 (9):795-804.

5. Bilgel M, et al. Individual estimates of age at detectable amyloid onset for risk factor assessment. Alzheimers Dement. 2016;12 (4):373-379.

6. Perrin RJ, Fagan AM, Holtzman DM. Multimodal techniques for diagnosis and prognosis of Alzheimer's disease. Nature. 2009;461 (7266):916-922

7. Klunk WE, et al. Imaging brain amyloid in Alzheimer's disease with Pittsburgh Compound-B. Ann Neurol. 2004;55 (3):306-319.

8. Blennow K, Mattsson N, Schöll M, Hansson O, Zetterberg H. Amyloid biomarkers in Alzheimer's disease. Trends Pharmacol Sci. 2015;36(5):297-309.

9. Khan TK, Alkon DL. Alzheimer's disease cerebrospinal fluid and neuroimaging biomarkers: diagnostic accuracy and relationship to drug efficacy. J Alzheimers Dis. 2015;46 (4):817-836.

10. Tu P, Fu H, Cui M. Compounds for imaging amyloid- $\beta$ deposits in an Alzheimer's brain: a patent review. Expert Opin Ther Pat. $2015 ; 25(4): 413-423$.

11. Hart NJ, Koronyo Y, Black KL, Koronyo-Hamaoui M. Ocular indicators of Alzheimer's: exploring disease in the retina. Acta Neuropathol. 2016;132 (6):767-787.

12. The Retina. In: Purves D, et al, eds. Neuroscience. 2nd ed. Chapters 11 and 12. Sunderland (MA): Sinauer Associates; 2001

13. Byerly MS, Blackshaw S. Vertebrate retina and hypothalamus development. Wiley Interdiscip Rev Syst Biol Med. 2009;1 (3):380-389.

14. Trost A, et al. Brain and Retinal Pericytes: Origin, Function and Role. Front Cell Neurosci. 2016;10:20.

15. Bayhan HA, Aslan Bayhan S, Celikbilek A, Tanık N, Gürdal C. Evaluation of the chorioretinal thickness changes in Alzheimer's disease using spectral-domain optical coherence tomography. Clin Experiment Ophthalmol. 2015;43 (2):145-151.

16. Blanks JC, Schmidt SY, Torigoe Y, Porrello KV, Hinton DR, Blanks RH. Retinal pathology in Alzheimer's disease. II. Regional neuron loss and glial changes in GCL. Neurobiol Aging. 1996;17(3):385-395.

17. Coppola G, et al. Optical coherence tomography in Alzheimer's disease: a meta-analysis. PLoS One. 2015;10(8):e0134750.

18. Curcio CA, Drucker DN. Retinal ganglion cells in Alzheimer's disease and aging. Ann Neurol. 1993;33 (3):248-257.

19. Feke GT, Hyman BT, Stern RA, Pasquale LR. Retinal blood flow in mild cognitive impairment and Alzheimer's disease. Alzheimers Dement (Amst). 2015;1 (2):144-151.

20. Frost S, et al. Retinal vascular biomarkers for early detection and monitoring of Alzheimer's disease. Transl Psychiatry. 2013;3:e233.

21. Gao L, Liu Y, Li X, Bai Q, Liu P. Abnormal retinal nerve fiber layer thickness and macula lutea in patients with mild cognitive impairment and Alzheimer's disease. Arch Gerontol Geriatr. 2015;60 (1):162-167.

22. Guo L, Duggan J, Cordeiro MF. Alzheimer's disease and retinal neurodegeneration. Curr Alzheimer Res. 2010;7(1):3-14.

23. Hinton DR, Sadun AA, Blanks JC, Miller CA. Optic-nerve degeneration in Alzheimer's disease. NEngl J Med. $1986 ; 315$ (8):485-487.

24. Kirbas S, Turkyilmaz K, Anlar O, Tufekci A, Durmus M. Retinal nerve fiber layer thickness in patients with Alzheimer disease. J Neuroophthalmol. 2013;33 (1):58-61.

25. Kromer R, Serbecic N, Hausner L, Froelich L, Aboul-Enein F, Beutelspacher SC. Detection of retinal nerve fiber layer defects in Alzheimer's disease Using SD-OCT. Front Psychiatry. 2014;5:22.

26. La Morgia C, et al. Melanopsin retinal ganglion cell loss in Alzheimer disease. Ann Neurol. 2016;79 (1):90-109.

27. Marziani E, et al. Evaluation of retinal nerve fiber layer and ganglion cell layer thickness in Alzheimer's disease using spectral-domain optical coherence tomography. Invest Ophthalmol Vis Sci. 2013;54 (9):5953-5958.

28. Moreno-Ramos T, Benito-León J, Villarejo A, Bermejo-Pareja F. Retinal nerve fiber layer thinning in dementia associated with Parkinson's disease, dementia with Lewy bodies, and Alzheimer's disease. J Alzheimers Dis. 2013;34 (3):659-664.

29. Moschos MM, et al. Structural and functional impairment of the retina and optic nerve in Alzheimer's disease. Curr Alzheimer Res. 2012;9 (7):782-788.

30. Paquet C, Boissonnot M, Roger F, Dighiero P, Gil R, Hugon J. Abnormal retinal thickness in patients with mild cognitive impairment and Alzheimer's disease. Neurosci Lett. 2007;420(2):97-99.

31. Shi Z, et al. Greater attenuation of retinal nerve fiber layer thickness in Alzheimer's disease patients. J Alzheimers Dis. 2014;40 (2):277-283.

32. Trick GL, Barris MC, Bickler-Bluth M. Abnormal pattern electroretinograms in patients with senile dementia of the Alzheimer type. Ann Neurol. 1989;26 (2):226-231.

33. Williams MA, et al. Retinal microvascular network attenuation in Alzheimer's disease. Alzheimers Dement (Amst). 2015;1 (2):229-235.

34. Patton N, Aslam T, Macgillivray T, Pattie A, Deary IJ, Dhillon B. Retinal vascular image analysis as a potential screening tool for cerebrovascular disease: a rationale based on homology between cerebral and retinal microvasculatures. $J$ Anat. 2005;206 (4):319-348.

35. Vecino E, Rodriguez FD, Ruzafa N, Pereiro X, Sharma SC. Glia-neuron interactions in the mammalian retina. Prog Retin Eye Res. 2016;51:1-40.

36. Morin PJ, et al. Amyloid precursor protein is synthesized by retinal ganglion cells, rapidly transported to the optic nerve plasma membrane and nerve terminals, and metabolized. J Neurochem. 1993;61 (2):464-473.

37. Cai J, et al. $\beta$-Secretase (BACE1) inhibition causes retinal pathology by vascular dysregulation and accumulation of age pigment. EMBO Mol Med. 2012;4 (9):980-991.

38. Li L, et al. BACE1 in the retina: a sensitive biomarker for monitoring early pathological changes in Alzheimer's disease. Neural Regen Res. 2016;11 (3):447-453.

39. Alexandrov PN, Pogue A, Bhattacharjee S, Lukiw WJ. Retinal amyloid peptides and complement factor $\mathrm{H}$ in transgenic models of Alzheimer's disease. Neuroreport. 2011;22 (12):623-627. 
40. Koronyo-Hamaoui M, et al. Identification of amyloid plaques in retinas from Alzheimer's patients and noninvasive in vivo optical imaging of retinal plaques in a mouse model. Neuroimage. 2011;54 Suppl 1:S204-S217.

41. Schön C, et al. Long-term in vivo imaging of fibrillar tau in the retina of P301S transgenic mice. PLoS ONE. 2012;7 (12):e53547. 42. Tsai Y, et al. Ocular changes in TgF344-AD rat model of Alzheimer's disease. Invest Ophthalmol Vis Sci. 2014;55 (1):523-534.

43. Ho CY, Troncoso JC, Knox D, Stark W, Eberhart CG. Beta-amyloid, phospho-tau and alpha-synuclein deposits similar to those in the brain are not identified in the eyes of Alzheimer's and Parkinson's disease patients. Brain Pathol. 2014;24 (1):25-32.

44. Ju YE, et al. Sleep quality and preclinical Alzheimer disease. JAMA Neurol. 2013;70 (5):587-593.

45. Du LY, et al. Alzheimer's disease-related protein expression in the retina of Octodon degus. PLoS ONE. 2015;10 (8):e0135499.

46. Dutescu RM, Li QX, Crowston J, Masters CL, Baird PN, Culvenor JG. Amyloid precursor protein processing and retinal pathology in mouse models of Alzheimer's disease. Graefes Arch Clin Exp Ophthalmol. 2009;247 (9):1213-1221.

47. Edwards MM, Rodríguez JJ, Gutierrez-Lanza R, Yates J, Verkhratsky A, Lutty GA. Retinal macroglia changes in a triple transgenic mouse model of Alzheimer's disease. Exp Eye Res. 2014;127:252-260.

48. Gao L, et al. Neuroprotective effect of memantine on the retinal ganglion cells of APPswe/PS1 $\triangle \mathrm{E} 9$ mice and its immunomodulatory mechanisms. Exp Eye Res. 2015;135:47-58.

49. Gupta VK, et al. Amyloid $\beta$ accumulation and inner retinal degenerative changes in Alzheimer's disease transgenic mouse. Neurosci Lett. 2016;623:52-56.

50. He Y, Zhao H, Su G. Ginsenoside Rg1 decreases neurofibrillary tangles accumulation in retina by regulating activities of neprilysin and PKA in retinal cells of AD mice model. J Mol Neurosci. 2014;52 (1):101-106.

51. Koronyo Y, Salumbides BC, Black KL, Koronyo-Hamaoui M. Alzheimer's disease in the retina: imaging retinal a $\beta$ plaques for early diagnosis and therapy assessment. Neurodegener Dis. 2012;10 (1-4):285-293.

52. Liu B, et al. Amyloid-peptide vaccinations reduce \{beta\}-amyloid plaques but exacerbate vascular deposition and inflammation in the retina of Alzheimer's transgenic mice. Am J Pathol. 2009;175 (5):2099-2110.

53. More SS, Vince R. Hyperspectral imaging signatures detect amyloidopathy in Alzheimer's mouse retina well before onset of cognitive decline. ACS Chem Neurosci. 2015;6 (2):306-315

54. Ning A, Cui J, To E, Ashe KH, Matsubara J. Amyloid-beta deposits lead to retinal degeneration in a mouse model of Alzheimer disease. Invest Ophthalmol Vis Sci. 2008;49 (11):5136-5143.

55. Park SW, et al. Intracellular amyloid beta alters the tight junction of retinal pigment epithelium in 5XFAD mice. Neurobiol Aging. 2014;35 (9):2013-2020.

56. Parthasarathy R, et al. Reduction of amyloid-beta levels in mouse eye tissues by intra-vitreally delivered neprilysin. Exp Eye Res. 2015;138:134-144.

57. Perez SE, Lumayag S, Kovacs B, Mufson EJ, Xu S. Beta-amyloid deposition and functional impairment in the retina of the APPswe/PS1DeltaE9 transgenic mouse model of Alzheimer's disease. Invest Ophthalmol Vis Sci. 2009;50 (2):793-800.

58. Pogue AI, Dua P, Hill JM, Lukiw WJ. Progressive inflammatory pathology in the retina of aluminum-fed 5xFAD transgenic mice. J Inorg Biochem. 2015;152:206-209.

59. Williams PA, et al. Retinal ganglion cell dendritic degeneration in a mouse model of Alzheimer's disease. Neurobiol Aging. 2013;34 (7):1799-1806.

60. Yang Y, et al. Suppressed retinal degeneration in aged wild type and APPswe/PS1 $\triangle \mathrm{E} 9$ mice by bone marrow transplantation. PLoS ONE. 2013;8 (6):e64246.

61. Zhao H, et al. Hyperphosphorylation of tau protein by calpain regulation in retina of Alzheimer's disease transgenic mouse. Neurosci Lett. 2013;551:12-16.

62. Kobayashi S, et al. Quantitative analysis of brain perfusion SPECT in Alzheimer's disease using a fully automated regional cerebral blood flow quantification software, 3DSRT. J Neurol Sci. 2008;264 (1-2):27-33.

63. Kumaraswamy P, Sethuraman S, Krishnan UM. Mechanistic insights of curcumin interactions with the core-recognition motif of $\beta$-amyloid peptide. J Agric Food Chem. 2013;61 (13):3278-3285.

64. Masuda Y, et al. Solid-state NMR analysis of interaction sites of curcumin and 42-residue amyloid $\beta$-protein fibrils. Bioorg Med Chem. 2011;19 (20):5967-5974.

65. Mutsuga $\mathrm{M}$, et al. Binding of curcumin to senile plaques and cerebral amyloid angiopathy in the aged brain of various animals and to neurofibrillary tangles in Alzheimer's brain. J Vet Med Sci. 2012;74 (1):51-57.

66. Yanagisawa D, et al. Relationship between the tautomeric structures of curcumin derivatives and their Abeta-binding activities in the context of therapies for Alzheimer's disease. Biomaterials. 2010;31 (14):4179-4185.

67. Yanagisawa D, Taguchi H, Yamamoto A, Shirai N, Hirao K, Tooyama I. Curcuminoid binds to amyloid- $\beta 1-42$ oligomer and fibril. J Alzheimers Dis. 2011;24 Suppl 2:33-42.

68. Ryu EK, Choe YS, Lee KH, Choi Y, Kim BT. Curcumin and dehydrozingerone derivatives: synthesis, radiolabeling, and evaluation for beta-amyloid plaque imaging. J Med Chem. 2006;49 (20):6111-6119.

69. Frautschy SA, Cole GM, Baird A. Phagocytosis and deposition of vascular beta-amyloid in rat brains injected with Alzheimer beta-amyloid. Am J Pathol. 1992;140 (6):1389-1399.

70. Ahmed M, et al. Structural conversion of neurotoxic amyloid-beta (1-42) oligomers to fibrils. Nat Struct Mol Biol. 2010;17 (5):561-567.

71. Walsh DM, et al. Amyloid beta-protein fibrillogenesis. Structure and biological activity of protofibrillar intermediates. $J$ Biol Chem. 1999;274(36):25945-25952.

72. Lashuel HA, Hartley D, Petre BM, Walz T, Lansbury PT. Neurodegenerative disease: amyloid pores from pathogenic mutations. Nature. 2002;418 (6895):291.

73. Teplow DB. On the subject of rigor in the study of amyloid $\beta$-protein assembly. Alzheimers Res Ther. 2013;5 (4):39.

74. Cox KH, Pipingas A, Scholey AB. Investigation of the effects of solid lipid curcumin on cognition and mood in a healthy older population. J Psychopharmacol (Oxford). 2015;29 (5):642-651.

75. DiSilvestro RA, Joseph E, Zhao S, Bomser J. Diverse effects of a low dose supplement of lipidated curcumin in healthy middle aged people. Nutr J. 2012;11:79.

76. Berisha F, Feke GT, Trempe CL, McMeel JW, Schepens CL. Retinal abnormalities in early Alzheimer's disease. Invest Ophthal- 
mol Vis Sci. 2007;48(5):2285-2289.

77. Kesler A, Vakhapova V, Korczyn AD, Naftaliev E, Neudorfer M. Retinal thickness in patients with mild cognitive impairment and Alzheimer's disease. Clin Neurol Neurosurg. 2011;113(7):523-526.

78. Lu Y, et al. Retinal nerve fiber layer structure abnormalities in early Alzheimer's disease: evidence in optical coherence tomography. Neurosci Lett. 2010;480 (1):69-72.

79. Trick GL, Trick LR, Morris P, Wolf M. Visual field loss in senile dementia of the Alzheimer's type. Neurology. 1995;45 (1):68-74.

80. Gilmore GC, Wenk HE, Naylor LA, Koss E. Motion perception and Alzheimer's disease. J Gerontol. 1994;49(2):P52-P57.

81. Risacher SL, et al. Visual contrast sensitivity in Alzheimer's disease, mild cognitive impairment, and older adults with cognitive complaints. Neurobiol Aging. 2013;34 (4):1133-1144.

82. Zuroff L, Daley D, Black KL, Koronyo-Hamaoui M. Clearance of cerebral A $\beta$ in Alzheimer's disease: reassessing the role of microglia and monocytes. Cell Mol Life Sci. 2017;74 (12):2167-2201.

83. Goldstein LE, et al. Cytosolic beta-amyloid deposition and supranuclear cataracts in lenses from people with Alzheimer's disease. Lancet. 2003;361 (9365):1258-1265.

84. Bodis-Wollner I, Kozlowski PB, Glazman S, Miri S. $\alpha$-synuclein in the inner retina in parkinson disease. Ann Neurol. 2014;75 (6):964-966.

85. Blanks JC, Hinton DR, Sadun AA, Miller CA. Retinal ganglion cell degeneration in Alzheimer's disease. Brain Res. 1989;501(2):364-372.

86. Katz B, Rimmer S, Iragui V, Katzman R. Abnormal pattern electroretinogram in Alzheimer's disease: evidence for retinal ganglion cell degeneration? Ann Neurol. 1989;26 (2):221-225.

87. Cordeiro MF, et al. Imaging multiple phases of neurodegeneration: a novel approach to assessing cell death in vivo. Cell Death Dis. 2010;1:e3.

88. Meli G, et al. Conformational targeting of intracellular A $\beta$ oligomers demonstrates their pathological oligomerization inside the endoplasmic reticulum. Nat Commun. 2014;5:3867.

89. Pensalfini A, et al. Intracellular amyloid and the neuronal origin of Alzheimer neuritic plaques. Neurobiol Dis. $2014 ; 71: 53-61$.

90. Armstrong RA. Beta-amyloid plaques: stages in life history or independent origin? Dement Geriatr Cogn Disord. 1998;9(4):227-238

91. Vinters HV. Cerebral amyloid angiopathy. A critical review. Stroke. 1987;18(2):311-324.

92. Frost S, Martins RN, Kanagasingam Y. Ocular biomarkers for early detection of Alzheimer's disease. J Alzheimers Dis 2010;22 (1):1-16.

93. Iadecola C. Neurovascular regulation in the normal brain and in Alzheimer's disease. Nat Rev Neurosci. 2004;5 (5):347-360.

94. Ruitenberg A, et al. Cerebral hypoperfusion and clinical onset of dementia: the Rotterdam Study. Ann Neurol. 2005;57 (6):789-794.

95. Zlokovic BV. Neurovascular pathways to neurodegeneration in Alzheimer's disease and other disorders. Nat Rev Neurosci. 2011;12 (12):723-738

96. Kimbrough IF, Robel S, Roberson ED, Sontheimer H. Vascular amyloidosis impairs the gliovascular unit in a mouse model of Alzheimer's disease. Brain. 2015;138 (Pt 12):3716-3733.

97. Yang F, et al. Curcumin inhibits formation of amyloid beta oligomers and fibrils, binds plaques, and reduces amyloid in vivo. $J$ Biol Chem. 2005;280 (7):5892-5901.

98. Bieschke J, Zhang Q, Powers ET, Lerner RA, Kelly JW. Oxidative metabolites accelerate Alzheimer's amyloidogenesis by a two-step mechanism, eliminating the requirement for nucleation. Biochemistry. 2005;44 (13):4977-4983.

99. Han M, et al. Age-related structural abnormalities in the human retina-choroid complex revealed by two-photon excited autofluorescence imaging. J Biomed Opt. 2007;12 (2):024012.

100. Schnell SA, Staines WA, Wessendorf MW. Reduction of lipofuscin-like autofluorescence in fluorescently labeled tissue. $J$ Histochem Cytochem. 1999;47 (6):719-730.

101. Maiti P, et al. A comparative study of dietary curcumin, nanocurcumin, and other classical amyloid-binding dyes for labeling and imaging of amyloid plaques in brain tissue of 5×-familial Alzheimer's disease mice. Histochem Cell Biol. 2016;146 (5):609-625

102. Rao PP, Mohamed T, Teckwani K, Tin G. Curcumin binding to beta amyloid: a computational study. Chem Biol Drug Des. 2015;86 (4):813-820.

103. Garcia-Alloza M, Borrelli LA, Rozkalne A, Hyman BT, Bacskai BJ. Curcumin labels amyloid pathology in vivo, disrupts existing plaques, and partially restores distorted neurites in an Alzheimer mouse model. J Neurochem. 2007;102 (4):1095-1104.

104.Dhillon N, et al. Phase II trial of curcumin in patients with advanced pancreatic cancer. Clin Cancer Res. $2008 ; 14$ (14):4491-4499.

105. Gota VS, Maru GB, Soni TG, Gandhi TR, Kochar N, Agarwal MG. Safety and pharmacokinetics of a solid lipid curcumin particle formulation in osteosarcoma patients and healthy volunteers. J Agric Food Chem. 2010;58 (4):2095-2099.

106. Dadhaniya P, et al. Safety assessment of a solid lipid curcumin particle preparation: acute and subchronic toxicity studies. Food Chem Toxicol. 2011;49 (8):1834-1842.

107. Kayabasi U, Sergott R, Rispoli M. Retinal examination for the diagnosis of Alzheimer's disease. J Ophthalmic Pathol. 2014;3(4):1-4.

108. Dentchev T, Milam AH, Lee VM, Trojanowski JQ, Dunaief JL. Amyloid-beta is found in drusen from some age-related macular degeneration retinas, but not in drusen from normal retinas. Mol Vis. 2003;9:184-190.

109. Johnson LV, Leitner WP, Rivest AJ, Staples MK, Radeke MJ, Anderson DH. The Alzheimer's A beta -peptide is deposited at sites of complement activation in pathologic deposits associated with aging and age-related macular degeneration. Proc Natl Acad Sci USA. 2002;99 (18):11830-11835.

110. Ratnayaka JA, Serpell LC, Lotery AJ. Dementia of the eye: the role of amyloid beta in retinal degeneration. Eye (Lond). 2015;29 (8):1013-1026.

111. Algvere PV, Kvanta A, Seregard S. Drusen maculopathy: a risk factor for visual deterioration. Acta Ophthalmol. 2016 ;94 (5):427-433.

112. Jack CR, et al. Brain $\beta$-amyloid load approaches a plateau. Neurology. 2013;80(10):890-896.

113. Begum AN, et al. Curcumin structure-function, bioavailability, and efficacy in models of neuroinflammation and Alzheimer's disease. J Pharmacol Exp Ther. 2008;326 (1):196-208. 